\title{
Newly Identified Electrically Coupled Neurons Support Development of the Drosophila Giant Fiber Model Circuit
}

\author{
DTyler Kennedy, ${ }^{1}$ and Kendal Broadie ${ }^{1,2,3}$
}

https://doi.org/10.1523/ENEURO.0346-18.2018

${ }^{1}$ Department of Biological Sciences, Vanderbilt University and Medical Center, Nashville, Tennessee 37235 ,

${ }^{2}$ Department of Cell and Developmental Biology, Vanderbilt University and Medical Center, Nashville, Tennessee

37235, and ${ }^{3}$ Vanderbilt Brain Institute, Vanderbilt University and Medical Center, Nashville, Tennessee 37235

\begin{abstract}
The Drosophila giant fiber (GF) escape circuit is an extensively studied model for neuron connectivity and function. Researchers have long taken advantage of the simple linear neuronal pathway, which begins at peripheral sensory modalities, travels through the central GF interneuron (GFI) to motor neurons, and terminates on wing/leg muscles. This circuit is more complex than it seems, however, as there exists a complex web of coupled neurons connected to the GFI that widely innervates the thoracic ganglion. Here, we define four new neuron clusters dye coupled to the central GFI, which we name GF coupled (GFC) 1-4. We identify new transgenic Gal4 drivers that express specifically in these neurons, and map both neuronal architecture and synaptic polarity. GFC1-4 share a central site of GFI connectivity, the inframedial bridge, where the neurons each form electrical synapses. Targeted apoptotic ablation of GFC1 reveals a key role for the proper development of the GF circuit, including the maintenance of GFI connectivity with upstream and downstream synaptic partners. GFC1 ablation frequently results in the loss of one GFI, which is always compensated for by contralateral innervation from a branch of the persisting GFI axon. Overall, this work reveals extensively coupled interconnectivity within the GF circuit, and the requirement of coupled neurons for circuit development. Identification of this large population of electrically coupled neurons in this classic model, and the ability to genetically manipulate these electrically synapsed neurons, expands the GF system capabilities for the nuanced, sophisticated circuit dissection necessary for deeper investigations into brain formation.
\end{abstract}

Key words: circuit map; electrical synapse; innexin

\section{Significance Statement}

Genetic model neural circuits with individually identifiable neurons help us to understand how nervous systems wire together during development, and then operate through coordinated chemical and electrical signaling. The Drosophila giant fiber circuit has long served as such a model, due to large neuron size, genetic malleability, and easily visualized behavioral output: a jump in response to a threat. This study unveils new members of this circuit, all of which synapse with the circuit at one site on the central giant fiber interneuron. We use new tools to identify and transgenically manipulate these neurons and show that these neurons are required for proper circuit development. This study provides a detailed circuit map for further dissection of neuronal connectivity and electrically coupled communication.

Received August 31, 2018; accepted November 12, 2018; First published November 26, 2018.

The authors declare no competing financial interests.
Author contributions: T.K. and K.B. designed research; T.K. performed research; T.K. analyzed data; T.K. and K.B. wrote the paper.

This work was supported by National Institutes of Health $(\mathrm{NIH})$ Grants 


\section{Introduction}

The Drosophila giant fiber (GF) circuit is particularly suitable for single-neuron resolution neurodevelopmental studies for a number of reasons, all related to its role as an escape response circuit (Allen et al., 2006; Boerner and Godenschwege, 2011). The need for rapid signal conduction from the senses through brain to muscles promoted the evolution of very large neurons throughout this circuit, facilitating their visualization and manipulation (Power, 1948; Borgen et al., 2017). This enlargement is most prominent in the long-distance GF interneuron (GFI), which consolidates sensory information in the brain and projects through the neck into the thoracic ganglion (TG) via giant axons (Allen et al., 1998; Pézier et al., 2014). To increase communication speed and fidelity between neurons, the GF circuitry uses mixed chemical and electrical synapses (Thomas and Wyman, 1984; Blagburn et al., 1999; Fayyazuddin et al., 2006). These electrical synapses, composed of the Shaking-B innexin, can pass small tracer dyes to identify coupled partner neurons (Phelan et al., 1996).

The GF circuit targets two large muscle sets used for rapid escape behavior, the tergotrochanteral muscle (TTM), which controls the legs for jumping, and the dorsal longitudinal muscle (DLM), which controls the wings (Tanouye and Wyman, 1980). The escape behavior is easily scored, and muscles are accessible to electrophysiological recordings, providing two outlets to study wholecircuit function (Martinez et al., 2007; Augustin et al., 2011; von Reyn et al., 2014). The GFI connects to the TTM via the tergotrochanteral motoneuron (TTMn) and to the DLM via the peripherally synapsing interneuron (PSI), which in turn synapses onto the dorsal longitudinal motoneuron (Tanouye and Wyman, 1980; Allen et al., 2006). While the GF circuit is reported to be quite simple, electrophoretic injections with small dyes make it clear that the GFI is actually part of a much larger circuit network of undescribed neurons (Boerner and Godenschwege, 2011; Enneking et al., 2013; Kennedy and Broadie, 2017).

This larger GF circuit should come as no surprise, as most classically studied circuits are continuously being updated to include new neurons, increasing the appreciation of the complexity and interconnectivity within the brain (Lin et al., 2016; Talay et al., 2017; Cande et al.,

MH-084989 (to K.B.), and NS-092250 and HD-007502 (to T.K.). Imaging was performed with the Vanderbilt University Cell Imaging Shared Resource, which was supported by NIH Grants CA-68485 and DK-20593.

We thank the Bloomington Drosophila Stock Center (Indiana University, Bloomington, IN) for numerous transgenic lines used in this study, and $\mathrm{Dr}$. Jonathan Bacon (University of Sussex, UK) for the gift of Shaking-B antibody. We also thank Randall Golovin and Dr. James Sears for comments on this manuscript.

Correspondence should be addressed to Kendal Broadie, Department of Biological Sciences, Vanderbilt University and Medical Center, Nashville, TN 37235. E-mail: kendal.broadie@vanderbilt.edu.

https://doi.org/10.1523/ENEURO.0346-18.2018

Copyright $\odot 2018$ Kennedy and Broadie

This is an open-access article distributed under the terms of the Creative Commons Attribution 4.0 International license, which permits unrestricted use, distribution and reproduction in any medium provided that the original work is properly attributed.
2018). Describing the wiring diagrams of classic circuits within model brains is important for understanding how local circuits accomplish processing tasks while also overriding or promoting behaviors controlled by separated but interconnected circuits (Gaudry and Kristan, 2009; Stensmyr et al., 2012; von Reyn et al., 2014). More complex model circuits can better help to answer questions about how circuits develop and evolve over time (Ward et al., 2015; Tosches, 2017). Combining GF circuit manipulability with the full complement of GFI-coupled neurons should enable robust new avenues for experimentation on how neurons select partners, determine synaptic strength, and regulate neighboring circuits.

In this study, we use neurobiotin (NB) dye injection to map previously uncharacterized GF-coupled (GFC) neurons. We take advantage of the FlyLight Gal4 library collection to identify transgenic drivers for the GFC neurons (Brand and Perrimon, 1993; Jenett et al., 2012). This approach defined four new GFI-coupled neuron clusters (i.e., GFC1-4) within the GF circuit, which we characterize for their architecture, neuronal polarity, and synaptic connectivity. We show that the inframedial bridge (IB; Allen et al., 1998) is the GFI site where all the GFC neurons come together to synapse with the circuit. We ablate GF neurons by transgenic expression of the apoptotic head involution defective (Hid) protein (Zhou et al., 1997) to find that GFC1 and PSI are required for proper GFI development. We also find GFI axons always compensate for the loss of their bilaterally symmetric partner through new contralateral innervation. Together, this work broadens the known GF circuit and opens new avenues for studying electrically coupled circuit development, function, and plasticity.

\section{Materials and Methods}

\section{Drosophila genetics}

All animals were maintained on a standard cornmeal/agar/ molasses Drosophila food diet in a $12 \mathrm{~h}$ light/dark cycling incubator at $25^{\circ} \mathrm{C}$. Timed-lay eggs were collected for $2-3 \mathrm{~d}$, and experimental animals were selected from rearing tubes 10-14 d later. The following Drosophila lines were used for genetic crosses: $w^{1118}$ (RRID:BDSC_3605); $w^{1118}$; P\{GMR78A06GAL4\}attP2 (Jenett et al., 2012; RRID:BDSC_46999); $w^{1118}$; P\{GMR73C07-GAL4\}attP2 (RRID:BDSC_46689); $w^{1118}$; P\{GMR24H07-GAL4\}attP2 (RRID:BDSC_49317); $w^{1118 ;}$ P\{GMR42A06-GAL4\}attP2 (RRID:BDSC_41245); $w^{1118}$; R10B11-p65.AD\}attP40 (Dionne et al., 2018; RRID: BDSC_68807); $w^{1118}$; P\{GMR14A06-GAL4.DBD\}attP2 (RRID: BDSC_68738); $w^{1118}, y^{1} ; 10 \mathrm{X}$ UAS-ivs-mCD8::GFP attP40 (Pfeiffer et al., 2010); UAS-hid.Z/CyO (Zhou et al., 1997; RRID: BDSC_65403); and $w^{1118}$; UAS-DenMark, UAS-syt::GFP (Zhang et al., 2002; Nicolai et al., 2010; RRID:BDSC_33064). Both females and males were used in this study, with sexspecific selection stated in figure legends. All genotypes were verified with visible markers.

\section{Dye iontophoresis}

GFI dye injections were performed similar to the previously published methods (Boerner and Godenschwege, 2011; Kennedy and Broadie, 2017). Briefly; glass elec- 
trodes (Kwik-Fil Borosilicate Glass 1B100F-4, World Precision Instruments) were pulled on a laser electrode puller (Model P-2000, Sutter Instrument) to $10 \mathrm{M} \Omega$ resistance (3 $\mathrm{M} \mathrm{KCl)}$. Electrodes were filled with $0.25 \%$ tetramethylrhodamine isothiocyanate (TRITC)-dextran (10 kDa; Life Technologies) and 7\% neurobiotin (Vector Laboratories; RRID:AB_2313575) in double-distilled $\mathrm{dH}_{2} \mathrm{O}$. Filled electrodes were placed on a silver chloride wire mounted on a PCS-5000 Micromanipulator (Burleigh). Animals in physiologic saline were cut along the dorsal midline to access the cervical connective (CC), at which electrodes were inserted into the GFI. A square-pulse stimulator (Grass S48, Astro-Med) provided $7.5100 \mathrm{~ms}$ pulses/s for $2 \mathrm{~min}$ with the $20 \mathrm{nA}$ injected current monitored by an AxoClamp2B Amplifier. A Digidata data acquisition system (1320A, Molecular Devices) was controlled with Clampex 9.2 software.

\section{Confocal imaging}

Brains were fixed for $30 \mathrm{~min}$ in $4 \%$ paraformaldehyde/ sucrose (Electron Microscopy Services) in PBS (pH 7.2; Life Technologies) and then rinsed $3 \times$ with PBS, and blocked for $1 \mathrm{~h}$ with $1 \%$ bovine serum albumin (BSA; Sigma-Aldrich) in PBST (PBS + 0.2\% Triton X-100; Thermo Fisher Scientific). Labels were diluted in PBST with $0.2 \%$ BSA. The following labels were used: streptavidin::Cy5 (1:20; Life Technologies); rabbit anti-ShakB (1:250; Phelan et al., 1996); rabbit antiGFP (1:2000; Abcam; RRID:AB_303395); FITC goat antiGFP (1:500; Abcam; RRID:AB_305635); rabbit anti-RFP (1: 500; Rockland; RRID:AB_2209751); Alexa Fluor 488conjugated donkey anti-goat (1:250; Thermo Fisher Scientific; RRID:AB_2534102); Alexa Fluor 488-conjugated donkey anti-rabbit (1:250; Thermo Fisher Scientific; RRID: AB_2556546); Alexa Fluor-568-conjugated donkey antirabbit (1:250; Thermo Fisher Scientific; RRID:AB_2534017); Alexa Fluor-647 conjugated donkey anti-rabbit (1:250; Thermo Fisher Scientific; RRID:AB_2536183); and Alexa Fluor-633-conjugated goat anti-rabbit (1:250; Thermo Fisher Scientific; RRID:AB_141419). Next, preparations were rinsed $3 \times$ for 30 min in PBST and $1 \times$ in PBS, and then were mounted on glass microscope slides (Probe On Plus $25 \times$ $75 \times 1.0 \mathrm{~mm}$, Thermo Fisher Scientific) in 2,2'-thiodiethanol (Sigma-Aldrich; Staudt et al., 2007). To prevent crushing, double-sided poster tape (Scotch) was placed on each side of the brains. Coverslips (catalog \#1.5H, Zeiss) were sealed with nail polish (Hard as Nails, Sally Hansen). Fluorescent images were collected using a Zeiss LSM 880 Confocal Microscope with an AiryScan module, which has increased lateral resolution $(161 \mathrm{~nm}$ ) and signal-to-noise ratio (Sivaguru et al., 2016). Images show maximum $Z$-stack projections under standard confocal mode, unless otherwise noted in the figure legends.

\section{Data analyses}

Data processing and image creation were performed with FIJl software (version 2; RRID:SCR_002285; Schindelin et al., 2012; Schneider et al., 2012). Neuronal models and movies were created using Imaris (version 9.2; RRID: SCR_007370).

\section{Results}

\section{The giant fiber circuit exhibits extensive dye-coupled connectivity}

Small gap junction-permeable dyes used to study the GF circuit have consistently revealed an extensive, but uncharacterized, network of dye-coupled neurons (Boerner and Godenschwege, 2011; Enneking et al., 2013; Kennedy and Broadie, 2017). To thoroughly study the architecture and properties of these neurons, we iontophoretically injected the GFI with the highly gap junction-permeable NB dye, and then labeled the brains post hoc with a streptavidinconjugated fluorophore (Huang et al., 1992). Consistent with previously published work, this intracellular dye injection reveals an extensive network of neurons dye coupled to the GFI (Fig. 1). This dye coupling is the direct result of gap junction connectivity, as eliminating gap junctions using shaking- $B$ mutants (shak ${ }^{2}$ ) prevents all NB dye transfer (data not shown; Blagburn et al., 1999; Kennedy and Broadie, 2017). A summary of this newly identified GF circuitry is shown in Figure 1.

Although there are a large number of dye-labeled processes widely distributed throughout the TG (Fig. 1A), all published GF circuit maps name only two GFI-coupled cells: (1) TTMn and (2) PSI (Fig. 1B, old circuit map). Here, we map and characterize all of the dye-coupled neurons whose projections we can trace back to an identifiable cell soma. We have named these neurons GFC followed by an identifying number (Fig. $1 A, B$ ). In this study, we report the characterization of four neuron clusters (GFC14 ), each of which represents a bilaterally symmetric set of two to seven neurons (Fig. 1B, new circuit map). The processes of these neurons contact the descending GFI axons and reach into all three TG segments (TG1-3), but do not cross into the brain or abdominal ganglion (AG). To understand how the GFCs integrate into the GF circuit, we began by obtaining selective genetic access to these neurons.

\section{Transgenic Gal4 drivers for newly identified giant fiber-coupled neurons}

To accurately map and manipulate the separate GFC neuron populations, we set forth to identify Gal4 drivers with highly specific expression for each GFC using two approaches. First, we conducted an in silico screen through the entire Janelia FlyLight library, which includes lines generated from the Vienna Tiles project (9436 lines; Jenett et al., 2012; Tirian and Dickson, 2017). Using images of the GFI dye-labeled circuit (Fig. 1A), we screened for matching GFP expression patterns (Fig. 2). We identified highly specific Gal4 drivers for GFC1 (78A06; Fig. 2A) and GFC2 (73C07; Fig. 2B), as well as less specific drivers for GFC3 and GFC4. Second, for cleaner GFC3 and GFC4 drivers, we used the recent automated Color-Depth Maximum Intensity Projection (MIP) tool for the Drosophila transgenic database (Otsuna et al., 2018). Using the less specific driver lines as templates to search this library, we screened for specific Gal4 drivers for GFC3 and GFC4. This complementary approach uncovered a highly specific driver for GFC3 (24H07; Fig. 2C) and a combined driver for GFC3/4 (42A06; Fig. 2D). During our search with 

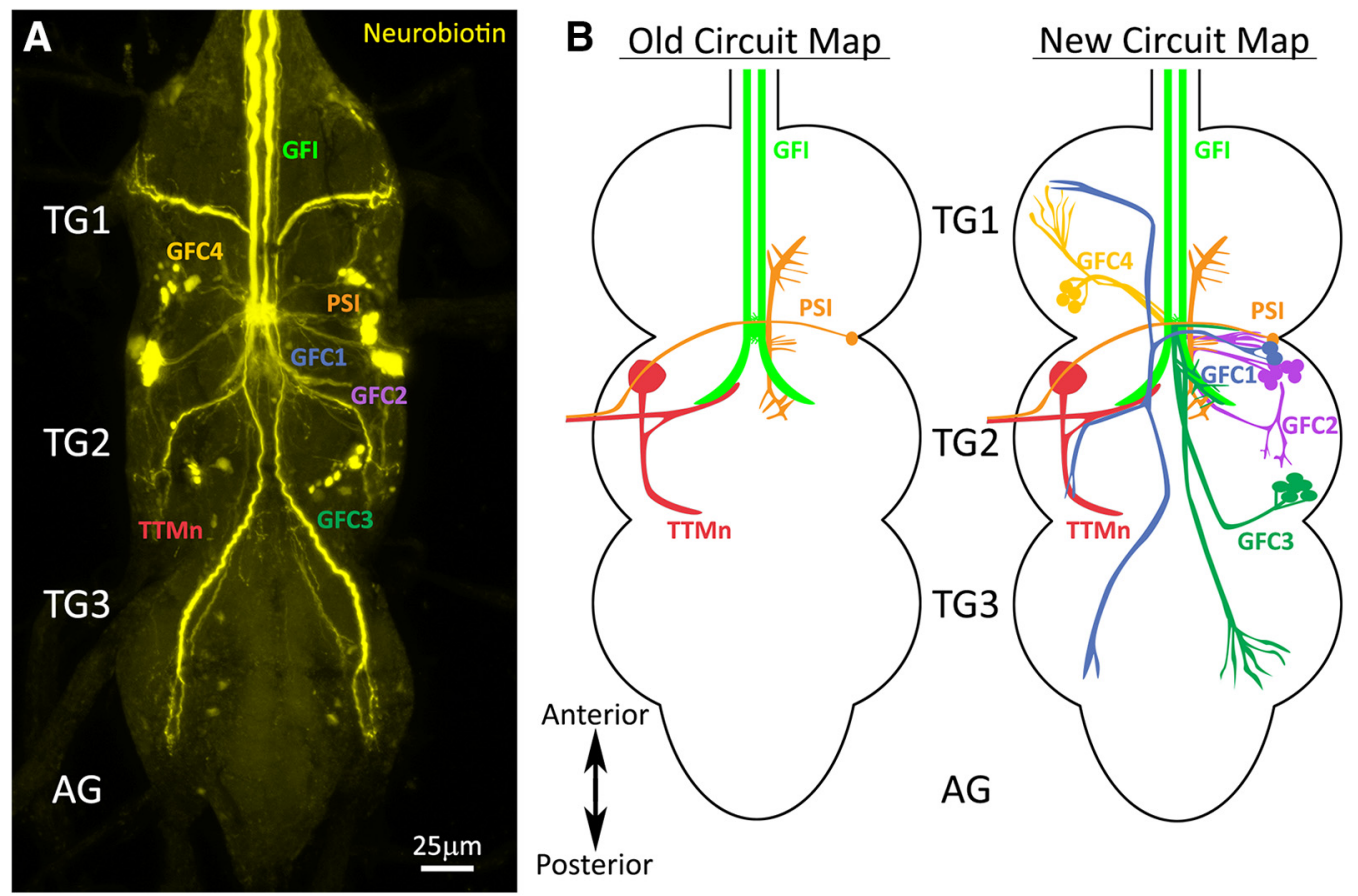

Figure 1. Giant fiber interneuron dye injection reveals coupled neurons. $\boldsymbol{A}$, The GFI iontophoretically injected with neurobiotin (yellow) shows extensive dye coupling to neurons in the TG. The established GFI-coupled neurons are (1) the PSI (orange) and (2) the TTMn (red). The newly identified GFCs project into all three TG segments (TG1-3), but do not extend into the AGs. B, Left, The old GF circuit map showing both of the previously characterized GFI (green) dye-coupled neurons: PSI (orange) and TTMn (red). Right, The new GF circuit map with the addition of all the newly identified GFC neurons from this study: GFC1 (blue), GFC2 (purple), GFC3 (dark green), and GFC4 (yellow).

the MIP tool, we identified many additional GF circuit drivers, aside from the ones used in this study. We selected the cleanest drivers and report them in Table 1 for use in future experiments.

To confirm that the new Gal4 transgenic driver lines label the bona fide GFC components of the GF circuit, we crossed each Gal4 line with the UAS-mCD8::GFP membrane reporter (Fig. 2, column 1) and injected the GFI with NB (Fig. 2, column 2). The merged images show perfect overlap between each transgenic driver line and the specified subset of the dye-labeled neurons (Fig. 2, column 3). Cell bodies are strongly labeled in all cases (Fig. 2, arrowheads), and individual neuronal processes of GFC1-4 can be traced for both the GFP and NB signals (Fig. 2, arrows). However, in some cases, such as GFC2 (73C07-Gal4), the dye injection signal is much dimmer than for other neurons, such as GFC1 (78A06-Gal4). Each GFC cluster is schematically represented within the TG, with full color on one side (Fig. 1, color scheme) and dashed outlines on the other side, to show each individual GFC neuron as well as their bilaterally symmetrical pattern (Fig. 2, column 4). Using these Gal4-driven GFP expression patterns, we are able to map each GFC cluster within the TG.

\section{Projection architecture of GFC neurons within the thoracic ganglion}

GFC1 is composed of two bilaterally symmetrical neurons on each side of TG2 (Fig. 2A). Each soma projects a process medially, which crosses the midline at the IB (Allen et al., 1998) and then splits, sending one branch anteriorly and one posteriorly. The anterior process travels halfway up TG1, then bends laterally and ventrally to terminate in the anterior corner of TG1, almost at the ventral-most point of the TG (Fig. $2 A$ ). This process extends several thin terminals, beginning in the same plane as the GFI bend. The posterior process splits halfway down TG2, just below the GFI bend. One branch proceeds laterally, then turns posteriorly toward the TG2 edge, with a ventral dive and several thin terminals, before terminating in the TG2 posterior lateral corner (Fig. 2A). The other process descends into TG3, bends inward toward the midline, then laterally to the anterior edge. From here, the process projects posteriorly and ventrally to end in a fashion similar to that of the other two terminals (Fig. 2A). All three GFC1 projections appear to innervate the leg neuropils (Namiki et al., 2018).

The seven bilaterally symmetric GFC2 neurons are largely restricted to TG2 (Fig. 2B). These cell bodies neighbor GFC1 and similarly project fasciculating processes medially. However, two-thirds of the way to the midline, the processes bend posteriorly and then laterally, to curve ventrally toward the lower edge of TG2 in the region of the GFI axon bend (Fig. $2 B$ ). The processes then curve anteriorly back toward the cell bodies, with a slight dorsal trajectory before termination, projecting several short, heavily branched termini in anterior and posterior directions. Another process doubles back toward the posterior deflection, travels medially to the midline and then sends out two branches posteriorly (Fig. 2B). One curves ventrolaterally to terminate along the first ventral 

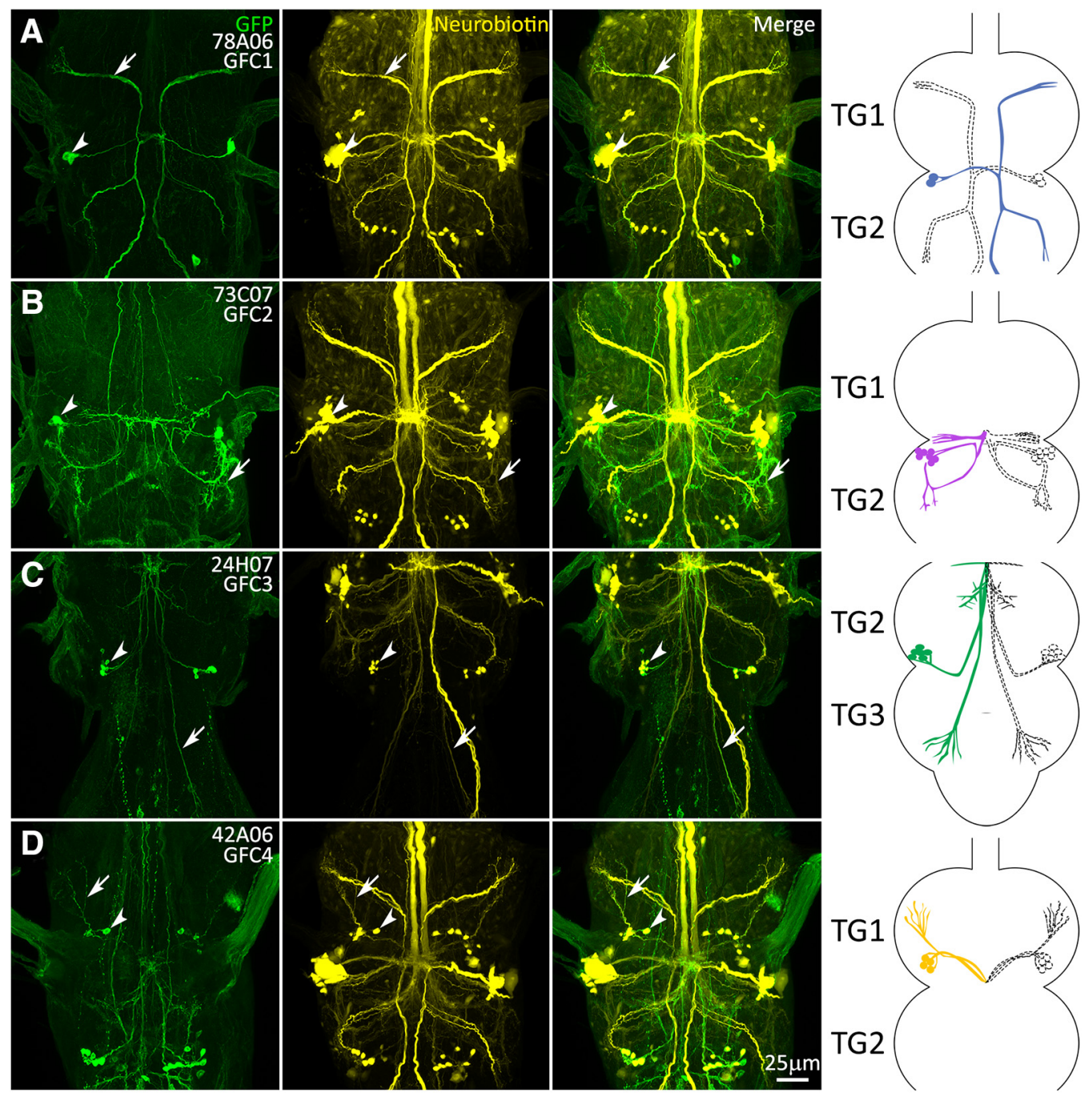

Figure 2. Transgenic Gal4 drivers for the newly identified GFC neurons. Gal4-driven expression of UAS-mCD8::GFP (green, column 1) overlapping with the GFI injection of neurobiotin dye (yellow, column 2) showing the identification of GFC drivers (merge, column 3). Arrows indicate processes with overlapping GFP and NB labeling, and arrowheads show the GFC cell bodies. The GFC neurons are drawn both in color (Fig. 1 color scheme) and perforated outlines to show their bilateral pattern (column 4). TG segments are selected to best show GFC projection architecture. All injections were performed on females. A, 78A06-Gal4 labels GFC1. The driver strength is relatively weak, with a somewhat stochastic labeling of the GFC1 neurons. B, 73C07-Gal4 labels GFC2. This driver is moderately strong, but also labels other neurons. C, 24H07-Gal4 labels GFC3. This driver strength is moderate, with labeling of other neurons. $\boldsymbol{D}, 42 \mathrm{A06}-\mathrm{GAL} 4$ labels both GFC3 and GFC4 neurons. The driver is relatively weak, with stochastic labeling of GFC4 neurons.

spiral, and the other travels dorsolaterally along the path of the original anterior process, terminating as it turns up toward the soma. There are two other processes that depart from the midline: one travels dorsally and slightly posteriorly before terminating, and one projects anteriorly and dorsolaterally to terminate in the lower central TG1
(Fig. 2B). These processes both appear to innervate the wing neuropils (Namiki et al., 2018).

GFC3 is composed of five bilaterally symmetrical neurons with the cell bodies positioned dorsally in the posterolateral corner of TG2 (Fig. 2C). These cells send out fasciculating processes that first proceed ventrally in a

Table 1: Transgenic Gal4 driver lines for the giant fiber circuit

\begin{tabular}{|c|c|c|c|c|c|c|}
\hline GFI & $\mathrm{GCl}$ & TTMn & PSI & GFC1 & GFC2 & GFC3 \\
\hline R14A01 & R32C04 & R25D08 & R26E04 & R93E07 & R13C08 & R44D02 \\
\hline VT042336 & VT002209 & VT038335 & VT030598 & VT059438 & VT043662 & R75D03 \\
\hline
\end{tabular}

New Gal4 drivers (distinct from those used in this study) that express selectively within the GF circuit, as compiled from the Janelia FlyLight and Vienna Tiles library collections. Selective lines for GFC4 have not been uncovered and thus are not reported here. 
medial-anterior direction up to the central IB connection with the GFI. At the IB, extensive GFC3 branches are visible, extending laterally and dorsally, but no further in either the anterior or ventral direction (Fig. 2C). These processes also track along the large terminal bend of the GFI axon. Dorsal to the IB, the main GFC3 processes reverse course to travel posterolaterally, while remaining ipsilateral to their cell bodies. The projection direction is ventral until TG3 is reached, at which point the processes move dorsally once again (Fig. $2 \mathrm{C}$ ). These processes terminate near the anterior portion of TG3 within the leg neuropil, in a series of thin processes at approximately the same axial plane as the IB and GFI axonal bends (Fig. 2C). Of note, both GFC1 and GFC3 were unintentionally captured in a recent screen for descending neurons (Namiki et al., 2018).

The four bilaterally symmetric GFC4 neurons are largely restricted to TG1 (Fig. 2D). The GFC4 cell bodies lie in the TG1 dorsal lateroposterior corner. The GFC4 processes first fasciculate to project ventrally, then posteromedially, running to the central IB (Fig. 2D). From the IB, the GFC4 processes then reverse course, remaining ipsilateral to their cell bodies as they project dorsally, back the way they came toward their cell bodies (Fig. 2D). When the GFC4 processes are directly below their cell bodies, they turn ventrally, and then travel toward the TG1 anterolateral corner to terminate in long finger-like projections (Fig. 2D). Like the other GFCs, the GFC4 processes appear to innervate the leg neuropils (Namiki et al., 2018). Overall, these transgenic driver lines allow detailed analysis of GFC architecture, and provide highly specific genetic control over the GFC neurons. To determine how these neurons interact with the GF circuit, we next examined their contact points with the GFI.

\section{The inframedial bridge connectivity site of GFI-GFC intersection}

GFC1-4 are all dye coupled to GFI via direct or indirect gap junction connections (Fig. 1), and all of these neurons project to the central IB to overlap with the GFI (Fig. 2). The IB has been defined as a region proximal to the GFI lateral axonal bend, where the GFI axon puts forth tufted projections and connects to the PSI (Allen et al., 1998). We therefore hypothesized that the IB is the primary site of GFI-GFC connectivity. To determine the location of potential synaptic sites between the GFI and GFCs, we injected the GFI with the large, nonpermeant dye TRITCdextran (10 kDa; Boerner and Godenschwege, 2011; Enneking et al., 2013; Kennedy and Broadie, 2017) for all the UAS-mCD8::GFP-labeled GFC1-4 lines (Fig. 3). We then assayed for overlap regions where the GFC membrane signal (Fig. 3, column 1) contacts the GFI TRITC signal (Fig. 3, column 2). Merging the two channels to create static (Fig. 3, column 3) and dynamic (Movies 1-4) 3D reconstructions of the spatial overlap provides clear identification of GFI-GFC contact points.

GFP and TRITC signals are color coded by depth to visualize the $Z$ dimension (FIJI plugin: Temporal-Color Code), with neurons proximal in $Z$ space displaying the same color (Fig. 3, columns 1 and 2). Overlap between neurons is shown for GFC neurons (Fig. 3, green) and GFI (Fig. 3, column 3, magenta). The results show that GFC1 contacts the GFI only at the IB with a simple crossing branch (Fig. 3A, arrows, Movie 1). GFC2-4 also contact the GFI directly at the IB, but with a much higher level of complexity (Fig. $3 B-D$, arrows, Movies 2-4). Further, GFC2 and GFC3 have processes that branch from the IB and overlap the large terminal bend of the GFI axon (Fig. 3B,C; arrowheads, Movies 2, 3). This is the first example, to our knowledge, of any contact along the GFI axonal bend other than TTMn. We also observe a third contact point between GFI and GFC3. The GFI axon bend occasionally extends small processes, which can contact GFC3 on posteriorly descending processes (Fig. 3C, arrowhead; magnified in inset). As these overlaps are likely sites for gap junction connectivity within the circuit, we investigated these membrane contacts for electrical synapses.

\section{Shaking-B gap junction synapses between GFI and GFC neurons}

The GF circuit is characterized by mixed chemical and electrical synapses (Blagburn et al., 1999; Allen et al., 2006). To map GFI-GFC electrical synapses, we labeled for the Shaking-B (ShakB) innexin, using an antibody recognizing the " $N+16$ " isoform present at GFI synapses (Phelan et al., 2008). Flies in which GFC1-4 neurons are labeled with UAS-mCD8::GFP (Fig. 4, column 1, green) were GFI injected with TRITC (Fig. 4, column 2, magenta) and colabeled with ShakB antibody (Fig. 4, column 3, cyan). All three channels were modeled with $3 \mathrm{D}$ rendering software to visualize ShakB-positive GFI-GFC contacts (Fig. 4, column 4, Movies 5-9). GFC1 (78A06-Gal4) exhibits a simple arborization, with a process coming across the $\mathrm{IB}$, and making a characteristic anterior-posterior split (Fig. 4A, Movie 5). ShakB is clearly visible in the 3D models, localized between the GFI and GFC1 as the process exits the IB (Fig. 4, arrows and inset). GFC1 projects axons to all three TG segments, indicating that there is a set of outputs triggered by the GFI escape response in parallel to TTM and DLM activation.

GFC2 neurons have a larger process field, forming a hemicircle in front of the GFI (Fig. 4B, Movie 6). Multiple ShakB electrical synapses clearly occur between the GFI and GFC2, although, due to the complexity of these connections, it is not possible to determine whether the GFI is contacting the GFC2 processes that come from the contralateral or ipsilateral sides of the TG, or both (Fig. 4B). GFC2 also contacts the GFI along the distal axonal bend (Fig. 3B), so we also investigated these sites for ShakB colocalization. The results show contact between the GFI and GFC2 near the tip of the bend; however, ShakB punctae are rarely seen colocalizing at these contacts (Fig. 4C, Movie 7), suggesting that these are primarily chemical synapse connections.

GFC3 has the most extensive IB contacts among all the GFCs, as well as broad interactions with surrounding neurons (Fig. 4D, Movie 8). GFC3 contacts the GFI with ShakB electrical synapses (Fig. $4 D$, arrows), but GFC3 branches extending beyond the IB are mostly ShakB 

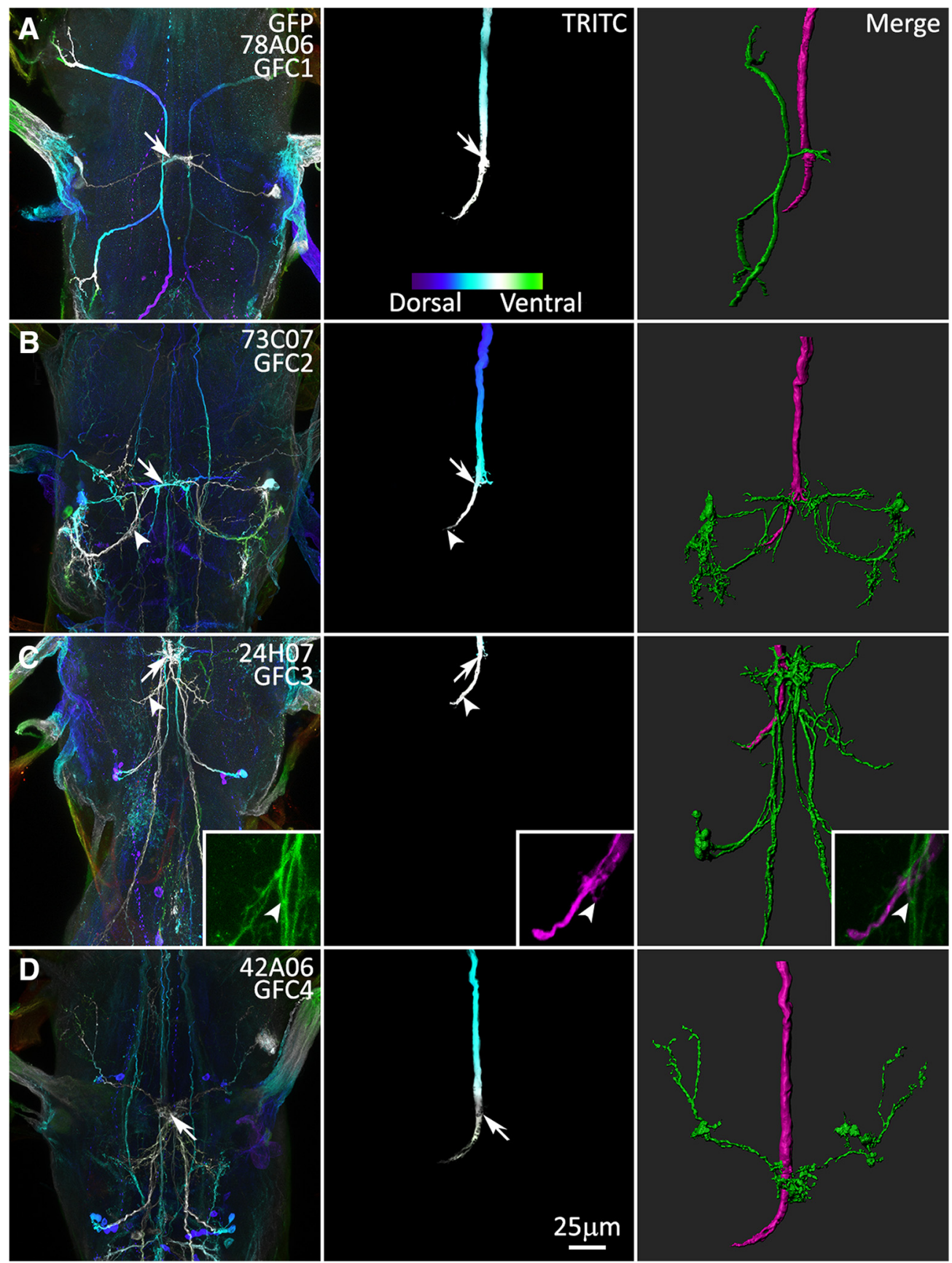

Figure 3. The GFI interacts with the GFC neurons at the inframedial bridge. Gal4 lines driving UAS- $m$ CD8::GFP (column 1) intersect with the GFI axon revealed by injection of TRITC (column 2), at the GFI IB and the GFI axonal bend (merge, column 3). The first two columns use depth color coding to represent the Z-position within the TG, with more dorsal regions displaying cool colors and ventral regions displaying warm colors (see color scale bar in $\boldsymbol{A}$, column 2). Arrows indicate overlapping membrane contact between GFCs and GFI at the IB. Arrowheads indicate GFC contact at the GFI axon bend. All injected flies are female. A, GFC1 (78A06-Gal4) interacts with the GFI exclusively at the IB. B, GFC2 (73C07-Gal4) interacts with the GFI at the IB and the GFI axonal bend. $\boldsymbol{C}$, GFC3 (24H07-Gal4) interacts with the GFI extensively at the IB and the GFI axonal bend. The GFI also produces small side projections that contact GFC3 (inset, arrowheads). D, GFC4 (42A06-Gal4) interacts with the GFI at the IB.

negative (Fig. 4D), indicating few electrical synapses. GFC3 contacts the GFI axon bend even more extensively than GFC2, but similarly has a small number of ShakB electrical synapse contacts (Fig. 4E, Movie 9). All images of GFI-GFC3 IB contact sites exhibit ShakB-positive electrical synapses, but only one image of the GFI-GFC3 axonal bend shows a synaptic connection (Fig. 4E, arrow). GFI axon bends are presynaptic to the TTMn, with extensive ShakB electrical synapses (Phelan et al., 2008), but it appears that only a small portion of this gap junction connectivity is used for GFC2 and GFC3, with the primary GFI-GFC electrical connections in the IB (Fig. 4B,D). With- 


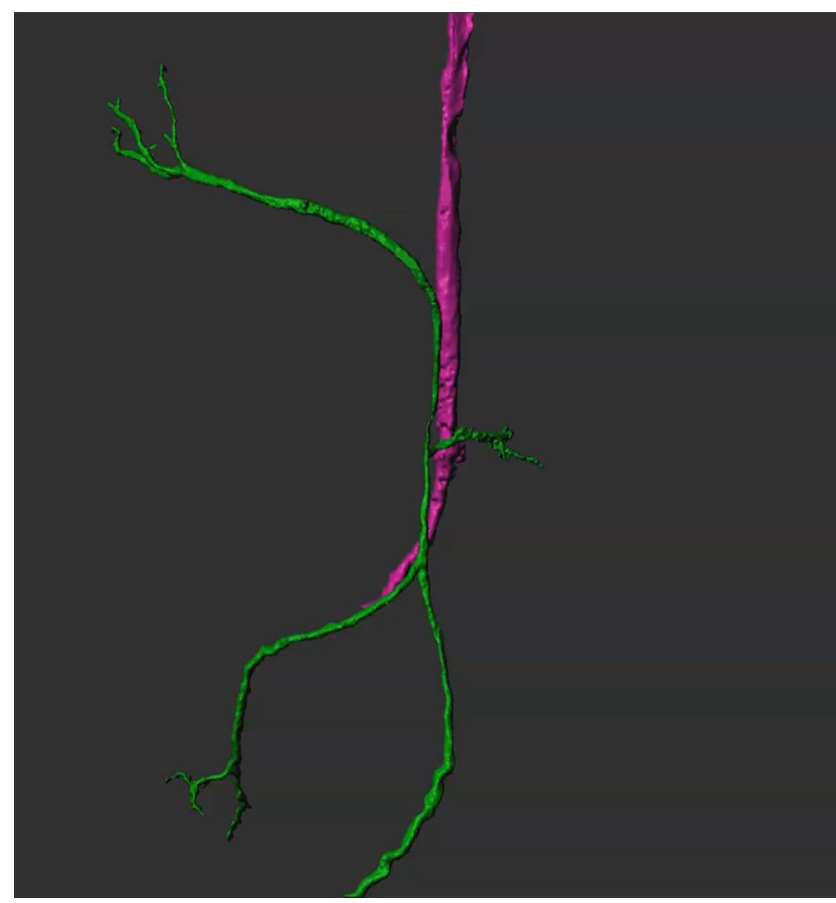

Movie 1. 3D animation of GFC1 and GFI interaction. Animated 3D reconstruction of mCD8::GFP-labeled GFC1 (green) and TRITC-injected GFI (magenta) in thoracic ganglion segments 1 and 2 (TG1/2). GFC1 intersects with the GFI in a narrow projection that crosses the IB. This projection then splits to create claw-like synaptic terminals in TG1-3 (TG3 not pictured). Scale bar, $20 \mu \mathrm{m}$. [View online]

out a GFC4-specific driver, we are unable to specifically test GFI-GFC4 ShakB synaptic connections. To determine the direction of information flow across GFI-GFC synapses, as well as connectivity in other regions of the TG, we next mapped the presynaptic and postsynaptic neuronal polarity of GFC1-4 synapses.

\section{Presynaptic and postsynaptic polarity of thoracic ganglion GFC neurons}

To investigate GFC postsynaptic domains, we used the UAS-DenMark dendrite reporter, composed of the exogenous mouse intercellular adhesion molecule- 5 dendritic protein fused to RFP (Nicolai et al., 2010). For presynaptic labeling, we used the UAS-synaptotagmin::GFP (Syt:: GFP) reporter, composed of the Syt1 integral synaptic vesicle protein fused to GFP (Zhang et al., 2002). In GFC1, the DenMark signal is absent from the finger-like projections at the process termini (Fig. $5 A$, column 1), and Syt::GFP is strongly present in a punctate array, indicating that these processes are presynaptic sites (Fig. 5A, column 2). In contrast, DenMark strongly labels GFC1 within the IB (Fig. 5A, arrow), indicating that this site is postsynaptic to the GFI (Fig. 5A, image column 3, top). The Syt::GFP signal is absent (Fig. $5 A$, image column 3 , bottom), suggesting that the IB site is solely for input. Together, these data indicate that GFC1 neurons receive presynaptic input into their dendrites at the IB and then project their contralateral axons for synaptic output into the leg neuropil (Namiki et al., 2018).

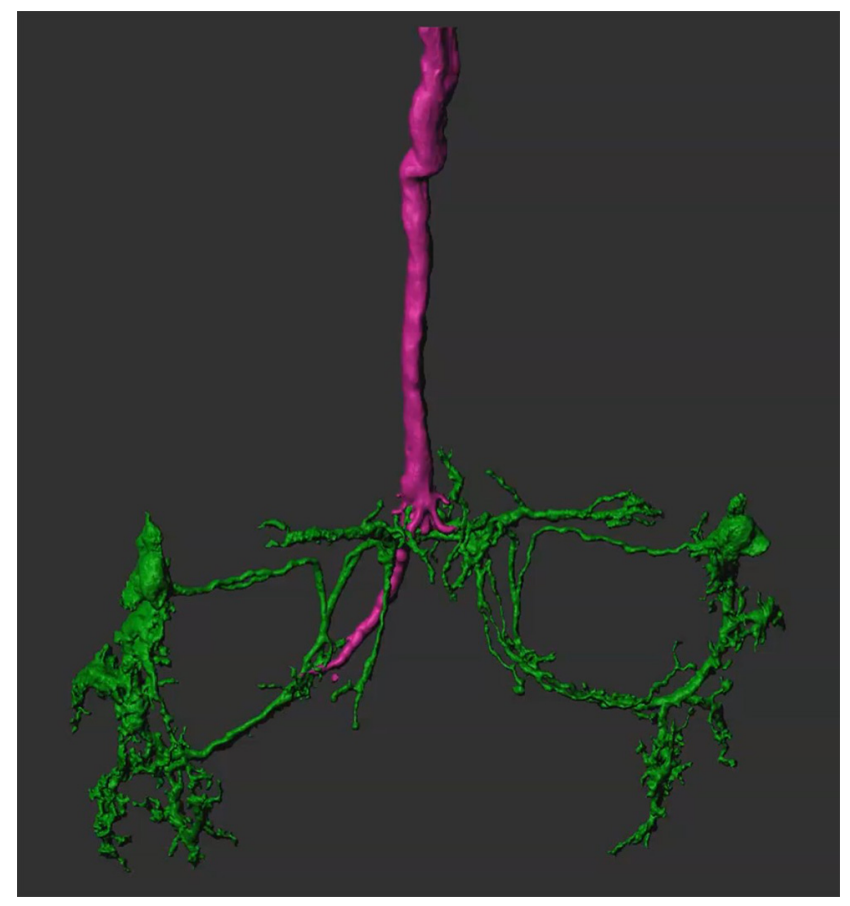

Movie 2. 3D animation of GFC2 and GFI interaction. Animated 3D reconstruction of mCD8::GFP-labeled GFC2 (green) and TRITC-injected GFI (magenta) in TG1/2. GFC2 extends a large TG2 loop with dorsal projections. GFC2 intersects with the GFI extensively at the IB and to a lesser extent at the tip of the TG2 axonal bend. Scale bar, $20 \mu \mathrm{m}$. [View online]

In contrast, GFC2 looped processes are strongly labeled by DenMark, including contacts at the GFI axon bend (Fig. 5B, column 1), with strongly colocalizing Syt:: GFP (Fig. 5B, column 2). Only the dorsolaterally projecting processes in the wing neuropil display Syt::GFP without DenMark present. Similarly within the IB, DenMark and Syt::GFP again colocalize, although DenMark is at a low level (Fig. 5B, image column 3). Thus, GFC2 neurons appear to have many colocalized presynaptic and postsynaptic domains. Note that it is not possible to tell where in the loop GFC2 processes double back, and the presynaptic and postsynaptic compartments may be in separate, adjacent processes (Fig. 5B). Based on our ShakB findings (Fig. 4B), it is likely that GFI and GFC2 directly synapse, but both appear presynaptic at the IB, and they may also share postsynaptic targets that mediate GFIGFC2 coupling. Another possibility is that GFI-GFC2 dye transfer does not occur at the IB, but instead they couple indirectly via an intermediary neuron. This could explain why the GFC2 is relatively poorly labeled by NB dye injection into the GFI, compared with other GFCs.

GFC3 has preynaptic and postsynaptic domains similar to GFC1 (Fig. 5C). The GFC3 long finger-like process projections in TG3 have a very weak DenMark signal (Fig. $5 C$, column 1) and very clear Syt::GFP punctae (Fig. 5C, column 2). Therefore, these sites are presumably presynaptic in leg neuropil (Namiki et al., 2018). At the IB, GFC3 strongly expresses DenMark (Fig. 5C, image column 3), which is thus postsynaptic. However, Denmark expression expands beyond the IB to include GFC3 branches 


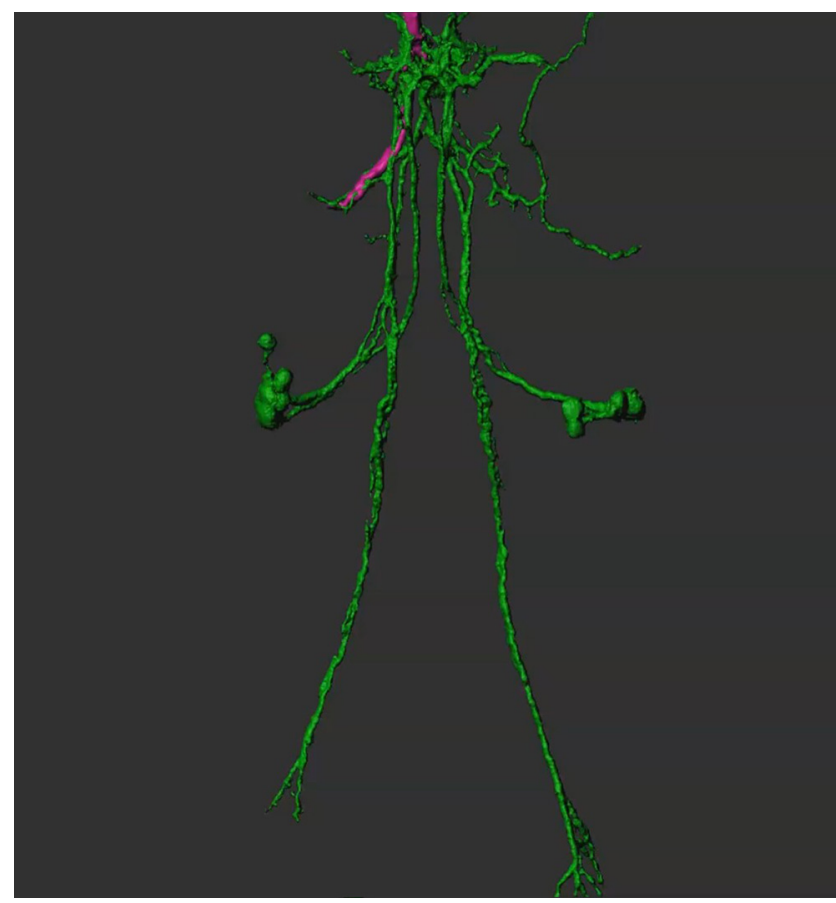

Movie 3. 3D animation of GFC3 and GFI interaction. Animated 3D reconstruction of mCD8::GFP-labeled GFC3 (green) and TRITC-injected GFI (magenta) in TG2/3. GFC3 cell bodies project processes to the IB and contact the GFI, with extensive branching, including along the GFI axonal bends. GFC3 then projects into TG3 to terminate. Scale bar, $20 \mu \mathrm{m}$. [View online]

that parallel the GFI axon bend and descending processes (Fig. 5C, arrowheads). Syt:GFP is undetectable at all of these GFC3 sites, indicating that they are solely postsynaptic (Fig. 5C, image column 3). Surprisingly, DenMark/Syt::GFP expression is lethal with the 42A06-Gal4 driver, and we were therefore unable to evaluate GFC4 presynaptic and postsynaptic domains. Based on similarities to GFC3, we predict that GFC4 has postsynaptic sites at the IB and presynaptic sites in the TG1 leg neuropil. Overall, DenMark and Syt::GFP clearly distinguish presynaptic and postsynaptic regions of all GFC neurons, except GFC2. As the GFCs are so intimately interconnected with the GFI, we next tested whether these coupled neurons play a role in GF circuit development or maintenance.

\section{GFC requirements for the development of GF circuit architecture}

We used Gal4-targeted expression of the Hid protein to drive apoptosis in GFC neurons, in an attempt to eliminate each GFC neuron and study the effects on the GF circuit architecture (Zhou et al., 1997; Muthukumar et al., 2014). Unfortunately, all of the GFC drivers used above (Fig. 2) are lethal in combination with UAS-hid. We repeated the study using split-Gal4 (spGal4) lines 10B11-AD $\cap$ 14A06DBD (Luan et al., 2006; Pfeiffer et al., 2010; Dionne et al., 2018) to eliminate the apoptosis of off-target cells. These spGal4 lines were identified using the MIP search tool and were selected for their strong expression in GFC1 with minimal overlap in nonspecific neurons. This spGal4 com-

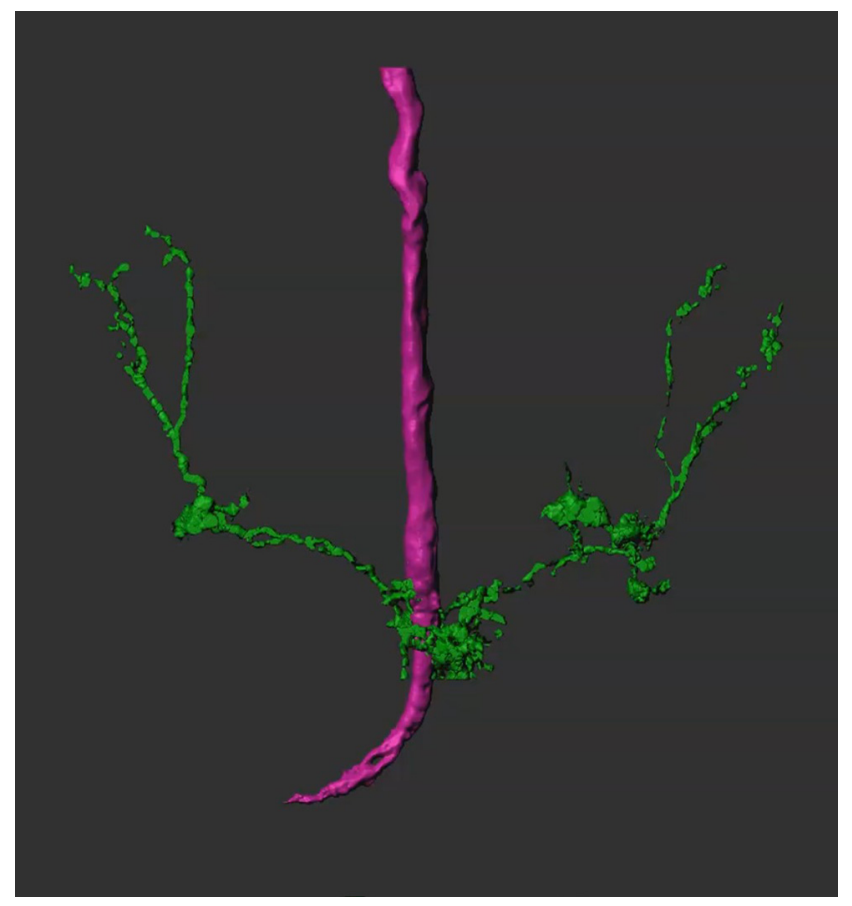

Movie 4. 3D animation of GFC4 and GFI interaction. Animated 3D reconstruction of mCD8::GFP-labeled GFC4 (green) and TRITC-injected GFI (magenta) in TG1/2. GFC4 cell bodies project processes from TG1 to the IB, then reverse course and return to TG1 where they terminate. Scale bar, $20 \mu \mathrm{m}$. [View online]

bination expresses strongly in GFC1, but also in PSI, as seen when crossed with UAS-mCD8::GFP (Fig. 6A, green) with injected TRITC (Fig. 6A, magenta) to label the GFI. In the brain (Fig. 6A, top), only TRITC dye is present in the GFI, where the GFI cell bodies (Fig. 6A, arrow) and their dendrites (arrowheads) reside. Importantly, no mCD8:: GFP is present in the GFI (Fig. 6A, green). Similarly, the giant commissural interneuron (GCl), which interconnects the GFls, displays no mCD8::GFP. In the TG, GFC1 (Fig. $6 A$, arrow) and PSI (Fig. $6 A$, arrowhead) express mCD8:: GFP (Fig. $6 A$, bottom).

NB dye injection into GFI in a UAS-hid/+ control animal shows both GFIs labeled in the brain (Fig. $6 B$, arrows). The $\mathrm{GCl}$ (Fig. 6B, arrowheads) interconnecting the GFI cell bodies (Allen et al., 1998) is also dye labeled. In the TG, the intact dye-coupled GF circuit is present in all UAShid/+ control animals (Fig. 6B, bottom). When the spGal4 driver is crossed to UAS-hid and the GFI injected with NB, GFC1 is ablated in 18 of 20 animals (90\%); fully in 14 of 20 animals, partially in 4 of 20 animals (Fig. 6C). Partial ablations are defined as several, but not all, neurons within GFC1 clusters being killed. PSI is eliminated in 16 of 20 animals (80\%). Two animals had no visible CC axons and could not be injected for analysis. The ablation of coupled cells causes stronger dye labeling in the persisting neurons, as expected due to the reduced volume of the GF circuit. As a consequence, the standard $2 \mathrm{~min}$ NB dye injection can cause lysis of the GF circuit, and therefore injection times were reduced to $\leq 30 \mathrm{~s}$ for these ablation experiments. This finding is similar to previous 


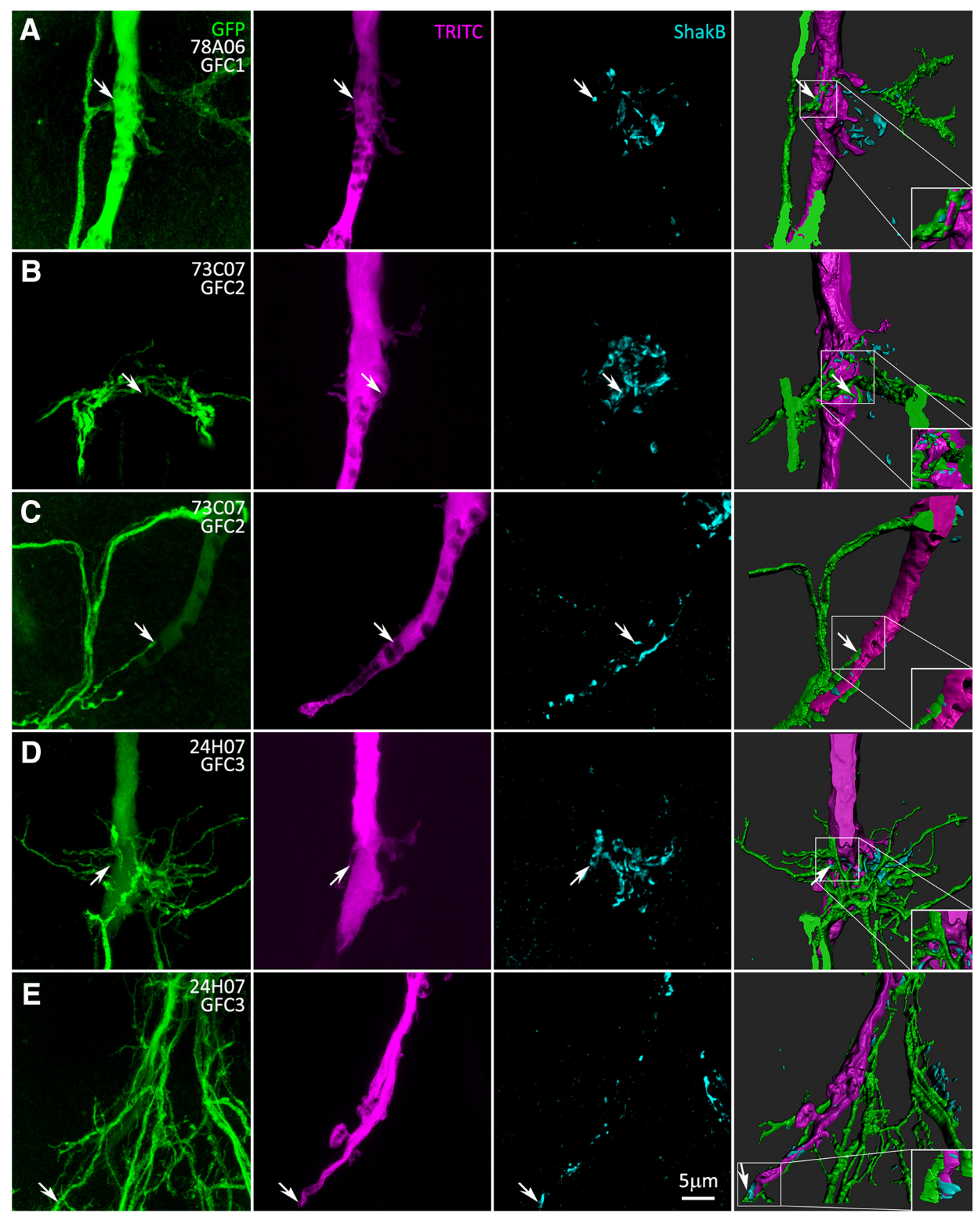

Figure 4. GFCs form electrical synapses with the GFI at the inframedial bridge. Electrical synapses between GFI and GFC neurons are shown in Gal4-driven UAS-mCD8::GFP animals (green, column 1) with TRITC dye injection into the GFI (magenta, column 2), while colabeling with the Shaking-B antibody (cyan, column 3). Images were taken using the AiryScan mode of the microscope. The three merged channels (column 4) show the regions of shared ShakB contact between GFI-GFCs. Arrows indicate sites of the GFI-GFC ShakB synaptic contacts (magnified in insets). All injected flies are female. A, GFC1 (78A06-Gal4) makes ShakB electrical synapse contacts with the GFI at the IB. B, GFC2 (73C07-Gal4) forms several ShakB electrical synapse contacts with the GFI. C, GFC2 (73C07-Gal4) contacts the GFI along the axonal bend. D, GFC3 (24H07-Gal4) contacts the GFI with multiple ShakB electrical synapses. E, GFC3 (24H07-Gal4) minimally contacts the GFI along the axonal bend (arrow).

reports when GFI dye coupling is eliminated through lack of interconnecting gap junctions (Kennedy and Broadie, 2017).

When testing the GF circuit for connectivity changes, we find GFC1/PSI ablation causes a striking impact on GFI development (Fig. 6C). All control animals (UAShid/+, $n=21$ ) display a completely normal dye-coupled GF circuit without detectable defects (Fig. 6B). With tar- geted UAS-hid ablation (spGal4 10B11-AD $\cap$ 14A06DBD>UAS-hid, $n=20$ animals), in 9 of 14 animals ( $\sim 65 \%$ ) with complete GFC1 ablation (including one case with the PSI present; Fig. 6C, arrowhead), one of the GFI neurons is completely absent (Fig. $6 D, E$ ). In partial GFC1 ablation cases, only one of four animals (25\%) lost a GFI. When a GFI is lost, there is no visible dye within the neuron, including the soma and the axon (Fig. 6C), and we 


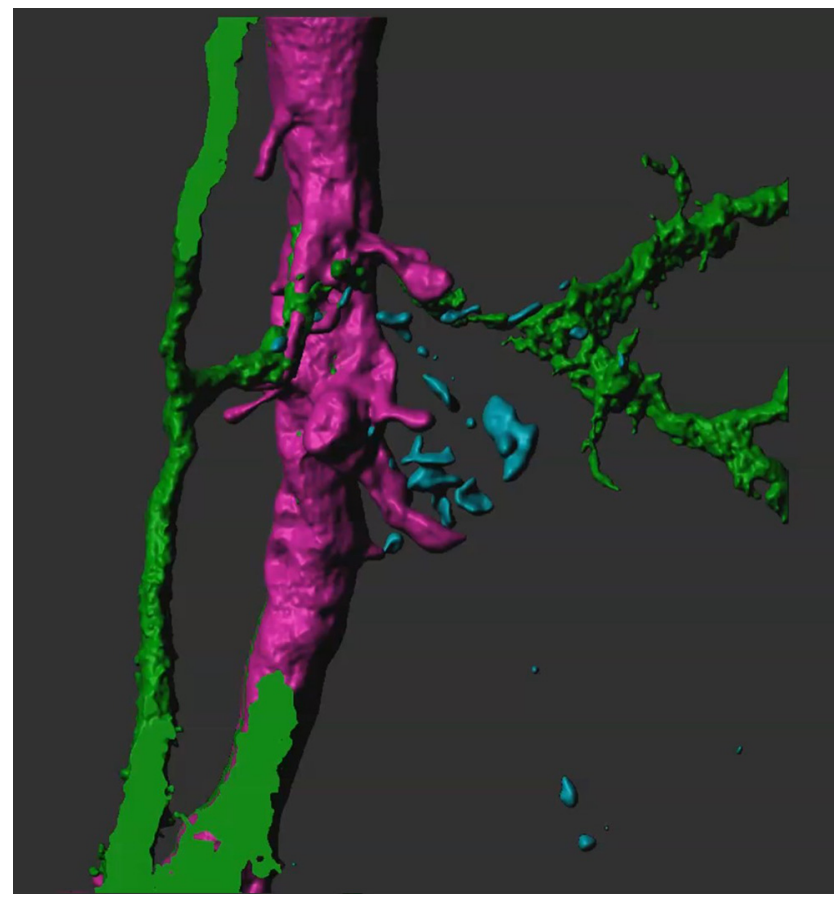

Movie 5. 3D animation of ShakB electrical synapses between GFC1 and GFI at IB. Animated 3D reconstruction of mCD8::GFPlabeled GFC1 (green), TRITC-injected GFI (magenta), and antiShakB electrical synapse labeling (cyan). The simple passing dendrite of GFC1 interacts with the GFI at multiple locations within the IB. Multiple sites of ShakB electrical synapses indicate direct GFC1-GFI coupling. Scale bar, $5 \mu \mathrm{m}$. [View online]

detect only one axon traveling through the CC by light microscopy. The remaining GFI always extends a compensating axon to the contralateral side (10 of 10 animals; $100 \%$ ) and forms a normal terminal axon bend (Fig. 6C, arrow).

Targeted UAS-hid expression is restricted to GFC1 and PS1, with no evidence of either GFI or GCl expression. A full summary of the experimental results is compared between UAS-hid/+ controls $(n=21)$ and the spGal4 10B11-AD $\cap$ 14A06-DBD>UAS-hid targeted ablation ( $n$ $=20$; Fig. $6 D, E$ ). Interestingly, in an animal with a fully intact GFC1 and only PSI ablation, both GFIs are present. In an animal with neither PSI nor GFC1 ablated, both GFIs are present (Fig. 6D,E). PSI ablation alone does not appear to be responsible for GFI loss, as GFI loss occurs when GFC1 alone is missing, but not when PSI alone is missing. We therefore conclude that GFC1 helps to maintain GFI during GF circuit development. Another interesting ablation result is the loss of $\mathrm{GFI}$ dye coupling to $\mathrm{GCl}$ in 5 of 10 animals (50\%) where a GFI is lost (Fig. 6C). Surprisingly, this loss of $\mathrm{GCl}$ also occurs in two animals where both GFIs are present; one with only GFC1 ablated, and the other with only PSI ablated. These results suggest the GFC neurons, alongside the classic GF circuit neurons, play an important role in neural circuit development.

\section{Discussion}

We describe here newly discovered neurons in the classic Drosophila GF neural circuit (Power, 1948; Sun

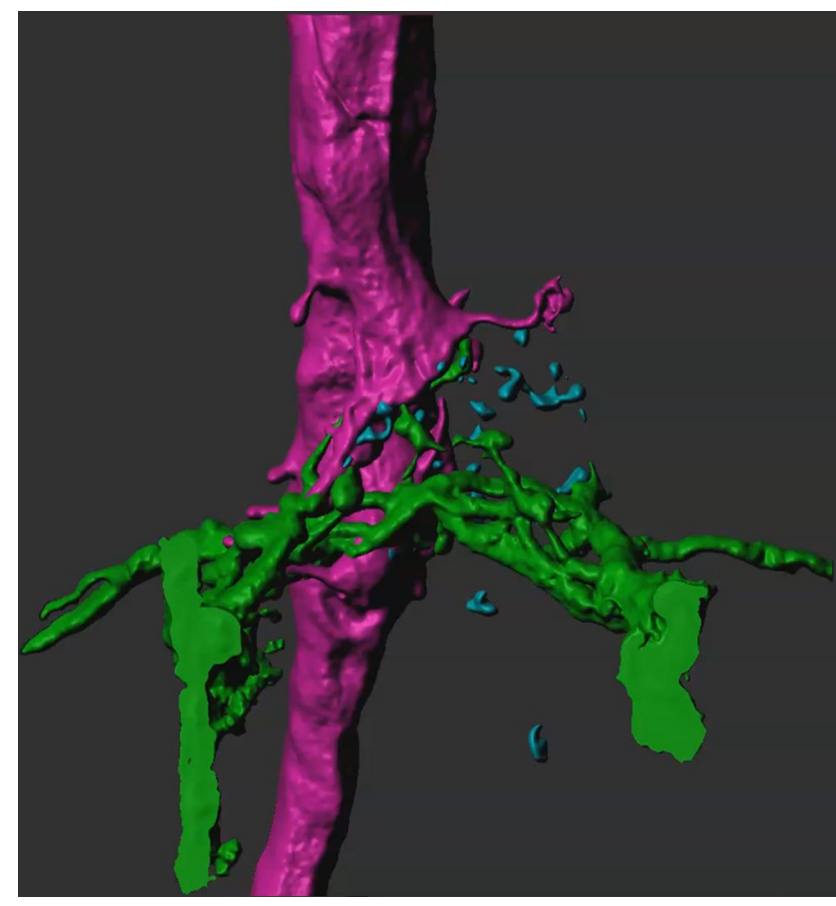

Movie 6. 3D animation of ShakB synapses between GFC3 and GFI at the axonal bend. Animated 3D reconstruction of mCD8::GFP-labeled GFC3 (green), TRITC-injected GFI (magenta), and anti-ShakB electrical synapse labeling (cyan). GFC3 extensively contacts the GFI along the GFI axonal bends in TG2. Despite this extensive contact, there are minimal ShakB punctae (cyan) shared between the neurons. Scale bar, $5 \mu \mathrm{m}$. [View online]

and Wyman, 1997; Jacobs et al., 2000; Allen et al., 2006) by characterizing four GFC neuron clusters. We identify specific transgenic drivers to both label and manipulate GFC1-4, and map neuronal architecture and polarity. We show that these neurons couple to the GFI via ShakB N + 16 innexin (Phelan et al., 2008) primarily at the central IB (Allen et al., 1998), but also at the downstream axonal bend. Alongside the already well established benefits of this circuit, including the large cell size, genetic malleability, and accessible functional/behavioral readouts (Power, 1948; Tanouye and Wyman, 1980; Phelan et al., 1996; Trimarchi et al., 1999), this expanded set of coupled neurons can aid future experiments in neurodevelopment, such as the study of axonal selection between multiple dendritic partners. This circuit map could be further refined using advanced tools, such as MultiColor FlpOut (Nern et al., 2015), as was recently accomplished for Drosophila brain descending neurons (Namiki et al., 2018).

This detailed circuit map is most useful for genetic analyses of electrical synapse partner connectivity between individually defined neurons. The GFCs identified in this study are composed of two to seven bilaterally symmetrical neurons clustered on each side of the TG segments. Similar clusters of repeated neurons with apparent connectivity redundancy have been recently identified in Drosophila brain descending neurons, where it is also unclear why neurons have such tightly overlapping pro- 


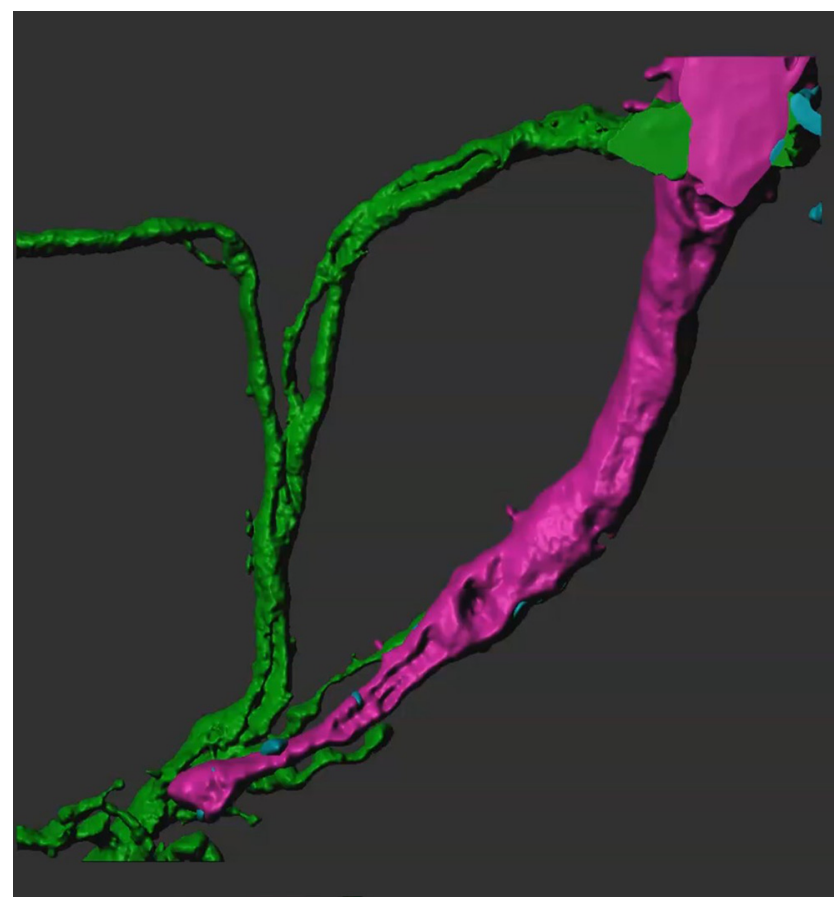

Movie 7. 3D animation of ShakB synapses between GFC2 and GFI at IB. Animated 3D reconstruction of mCD8::GFP-labeled GFC2 (green), TRITC-injected GFI (magenta), and anti-ShakB electrical synapse labeling (cyan). The GFC2 field interacts in multiple locations with the GFI, including several side projections from the IB. Several sites of ShakB electrical synapses indicate GFC2-GFI coupling. Scale bar, $5 \mu \mathrm{m}$. [View online]

jection patterns (Namiki et al., 2018). We have insufficient resolution to determine whether the GFC neurons truly are duplicates, or whether they have distinct, proximally adjacent synaptic targets, like the closely overlapping Kenyon cells of the adult brain mushroom body (Crittenden et al., 1998). It has been proposed that neuron duplication may allow for a sliding scale of response within a circuit, whereby more neurons are activated to increase the strength of the response (Namiki et al., 2018). Alternatively, if the neurons contact similar proximal synaptic targets, their role may be to provide ultrafine control of muscle movement in the GF circuit escape response (Namiki et al., 2018).

Complex leg and wing movements are thought to be controlled by extensive TG neural circuits, which are activated by a small number of descending neurons, including the GFI dedicated to rapid escape behavior (Cardona et al., 2009; Hsu and Bhandawat, 2016; Cande et al., 2018; Namiki et al., 2018). The roles of GFC neurons uncovered here have yet to be elucidated, although their electrical coupling to the GFI strongly suggests a close relationship to behaviors promoting or otherwise facilitating the rapid escape jump-and-flight response. Our preliminary attempts to optogenetically activate the GFC neurons through blue-light stimulation of Gal4-targeted ChR2-H134R (Nagel et al., 2005) or ChOP-XXL (Dawydow et al., 2014) channels did not produce behaviors. We suspect the stimulation paradigm was not strong enough, that appropriate sensory costimulation conditions may

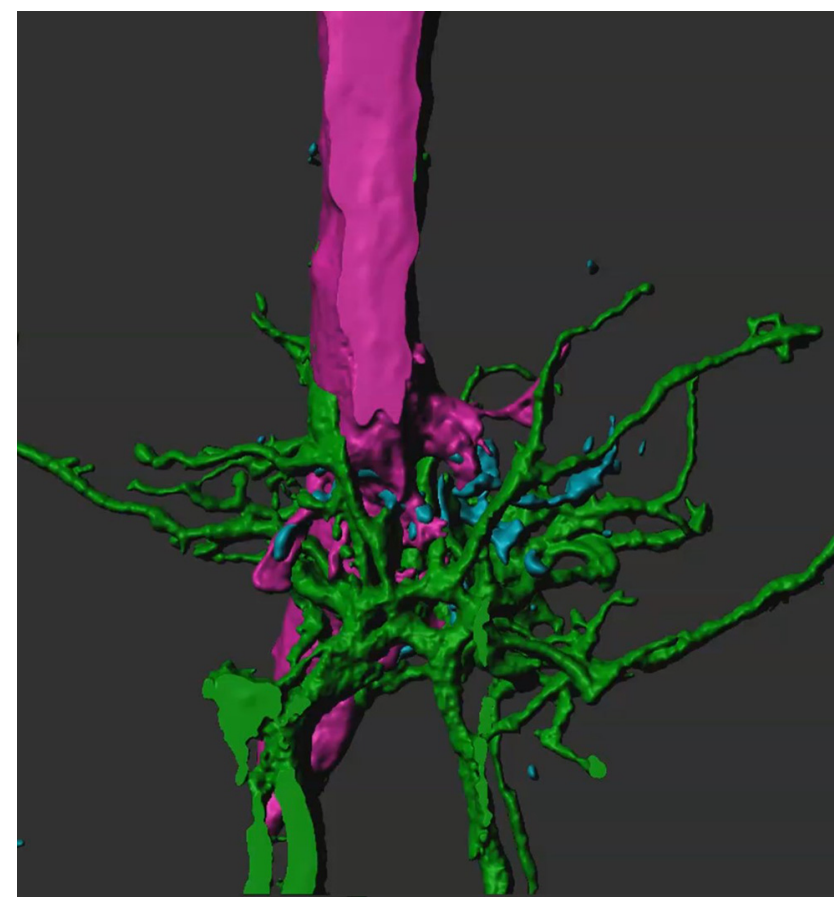

Movie 8. 3D animation of ShakB synapses between GFC2 and GFI at axonal bend. Animated 3D reconstruction of mCD8::GFPlabeled GFC2 (green), TRITC-injected GFI (magenta), and antiShakB electrical synapse labeling (cyan). GFC2 contacts the GFI along the TG2 axonal bends, mostly at the tips. Along these contact sites, there are few to no ShakB contacts (cyan) shared between the neurons. Scale bar, $5 \mu \mathrm{m}$. [View online]

not have been provided (von Reyn et al., 2014), that behavioral scoring methods were not sensitive enough to detect subtle motor output changes (Cande et al., 2018), or that these neurons modulate internal processes not directly manifest in rapid escape behavior (Joseph et al., 2017).

Based on the very recently proposed ventral nerve cord regional map (Namiki et al., 2018), the most likely targets of the four GFCs identified here are the TG1-3 leg neuropils. GFC2 also appears to target the TG2 wing neuropil. Both leg and wing outputs are integral to the GF circuit escape response (von Reyn et al., 2014). GFC1 targets all three TG leg neuropil segments; GFC2 targets TG2; and GFC3 and GFC4 target TG3 and TG1, respectively. This extensive leg neuropil connectivity may regulate tension in the front and hind legs, allowing the central legs to execute a more effective escape jump (Trimarchi and Schneiderman, 1993; von Reyn et al., 2014; Namiki et al., 2018). In support of this hypothesis, our work indicates that GFCs $1-3$ are all directly gap junction coupled to the descending $\mathrm{GFI}$, receiving input primarily at the IB, and thus share in the rapid conduction speed of the GF circuit (Phelan et al., 2008). Further, GFC3 neurons extend postsynaptic processes that parallel the PSI processes, indicating GFC3 may collect input from multiple neurons in the GF circuit.

Like the PSI, all four GFCs appear to synapse on their downstream targets via only chemical synapses, based on Syt::GFP synaptic vesicle marker and lack of ShakB 


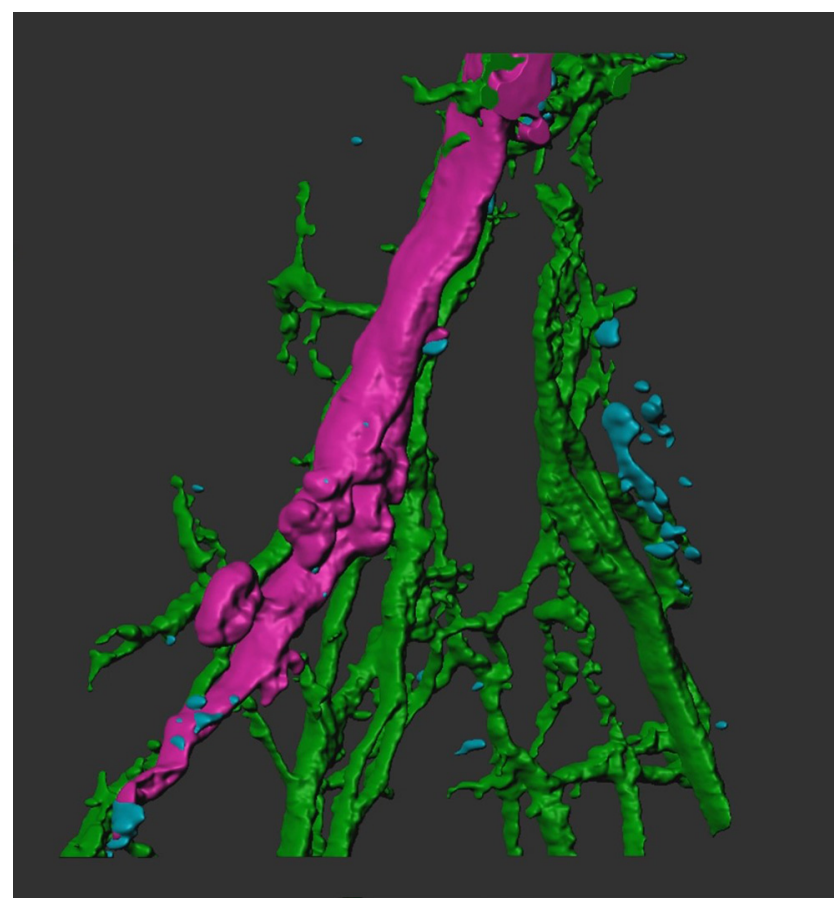

Movie 9. 3D animation of ShakB synapses between GFC3 and GFI at IB. Animated 3D reconstruction of mCD8::GFP-labeled GFC3 (green), TRITC-injected GFI (magenta), and anti-ShakB electrical synapse labeling (cyan). GFC3 extends the largest dendritic field at the IB, with extensive GFC3-GFI contact. Several of these contact points are positive for ShakB electrical synapses. Scale bar, $5 \mu \mathrm{m}$. [View online]

electrical synapse labeling at GFC termini (Allen et al., 2006). It might appear possible that another innexin could mediate these GFC connections (Stebbings et al., 2002; Phelan, 2005); however, the complete absence of dye coupling to neurons downstream of GFCs indicates electrical synapses are absent. In contrast to the other GFCs, GFC2 appears both presynaptic and postsynaptic at the IB connectivity hub, suggesting that it may share postsynaptic partners with GFI, potentially including GFC1, 3, and 4 and/or PSI. Given this circuit connectivity, GFC2 may trigger the rapid escape jump reflex independently of the GFI, in a parallel circuit output long speculated to exist, but not previously identified (Trimarchi and Schneiderman, 1995; Fotowat et al., 2009). Indeed, GFC2 extends presynaptic processes into the tergotrochanteral motoneuron dendritic field, thus mimicking GFI connectivity (King and Wyman, 1980).

DenMark and Syt::GFP reporters are extremely useful in defining neuron polarity (Zhang et al., 2002; Nicolai et al., 2010; Bidaye et al., 2014; Frank et al., 2015), but they have limitations that can make interpretation difficult. Both reporters preferentially mark appropriate synaptic regions, but can mislocalize due to transgenic overexpression (Chen et al., 2014; Kanca et al., 2017). A likely example here is dim DenMark signal near bright Syt::GFP punctae (Fig. 5C). The DenMark signal-to-noise ratio is much worse than the IB labeling, while the Syt::GFP signal-to-noise ratio is much stronger; hence, our conclusion that this region is presynaptic. A more problematic example may be the DenMark/Syt::GFP overlap in GFC2 (Fig. 5B). This labeling likely shows adjacent presynaptic and postsynaptic processes, which we cannot distinguish; although shared compartments have been reported in mushroom body Kenyon cells (Christiansen et al., 2011; Zheng et al., 2018). It is also worth noting that the 73C07-Gal4 line for GFC2 is the strongest driver used and may cause DenMark or Syt::GFP mislocalization via transgenic overexpression (Chen et al., 2014; Kanca et al., 2017). The 42A06-Gal4 driver for GFC3/4 is lethal with UAS-DenMark, syt::GFP, showing that these markers can also have detrimental effects.

Our targeted ablation studies indicate a role for GFCs in GF circuit development, and demonstrate the ability of the circuit to compensate for the loss of a GFI, much like ocular dominance columns in the classic work by Hubel and Wiesel (1970) and Hubel et al. (1977). PSI ablation does not appear to be responsible for the GFI loss, based on the fact that GFIs are present when PSI alone is ablated, and GFls are lost only when GFC1 is ablated. Another impact of ablation is lost $\mathrm{GCl}$ coupling when a GFI, GFC1, or PSI is removed. As $\mathrm{GCl}$ coupling loss occurs both when GFC1 alone is lost and when PSI alone is lost, it appears that complete GF circuit formation depends on feedback from multiple circuit members (Kandler and Katz, 1995; Hanganu et al., 2009; Maher et al., 2009; Belousov and Fontes, 2013). This finding suggests neurons not directly coupled can feedback through an intermediary circuit neuron; an intriguing but poorly studied hypothesis (Kandler and Katz, 1995; Belousov and Fontes, 2013). We note that the TTMn only occasionally dye couples with GFI, suggesting gap junction transitions between open and closed states could also contribute.

Previous studies have shown ablation of the GFI using neurotoxins, such as ricin (Smith et al., 1996), and have even found that single GFIs are lost at very low frequency in wild-type animals (Allen et al., 1998). In the latter case, the authors also found midline crossing of a compensatory contralateral process from the enduring GFI, as in our work. We hypothesize that the GFI loss reported here results from lost GFI stabilization by GFC1 due to the loss of trophic/synaptic signaling or physical contact (Gorin and Johnson, 1979; Pearson and Stoffler, 1992; Antonini and Stryker, 1993; Crowley et al., 1994; Uesaka, 2005; Gibson and Ma, 2011). Other GFI postsynaptic targets (PSI, TTMn, GFC2-4) presumably also participate in GFI stabilization, although Gal4 drivers tested thus far for these neurons have proved lethal in combination with UAS-hid (Zhou et al., 1997; Muthukumar et al., 2014). These animals die early in development, showing the need for spGal4 lines capable of avoiding off-target cells. Pursuing this phenotype with more specific drivers and screening approaches could elucidate molecular mechanisms that these neurons use to stabilize synaptic partners (Cohen-Cory, 2002).

Other methods shown to cause GFI axonal retraction and neuronal loss include blocking membrane endocytosis (e.g., using dominant-negative shibire/Dynamin) and the overexpression of select transmembrane receptors, 


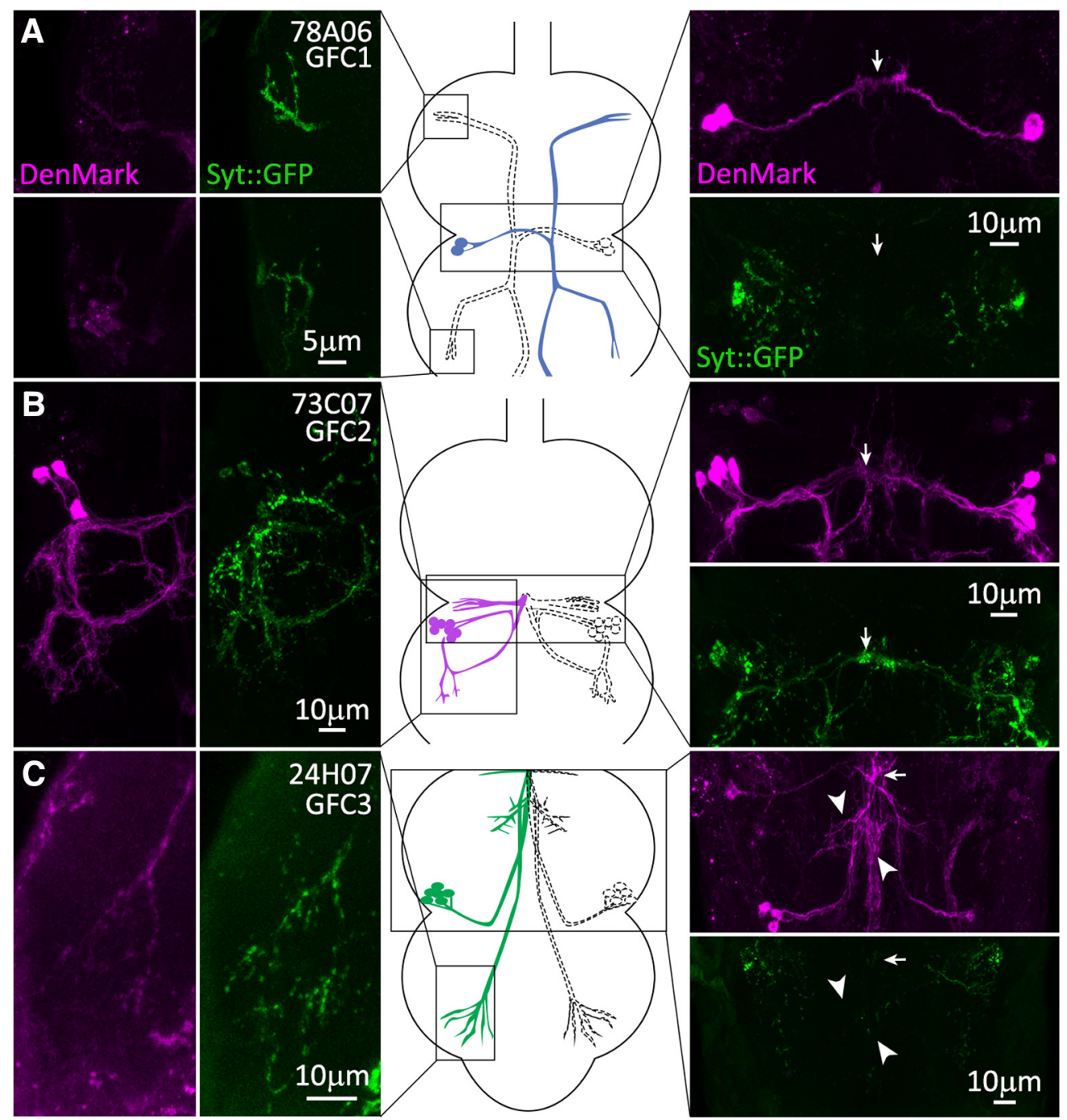

Figure 5. Presynaptic and postsynaptic polarity of the newly identified GFC neurons. GFC neuronal polarity is shown using the dendrite/soma label DenMark (magenta) and the presynaptic label synaptotagmin::GFP (Syt::GFP, green). Substacks of the regions of interest for each GFC are shown for DenMark (column 1) and Syt::GFP (column 2), with above and below paired comparisons (image column 3). Arrows indicate the position of the IB. GFC schematic representations are shown (center column), with regions of interest outlined in black boxes. A, GFC1 (78A06-Gal4) processes are labeled by presynaptic Syt::GFP in both TG1 (top) and TG2 (bottom) segments, while the IB is labeled by postsynaptic DenMark. B, GFC2 (73C07-Gal4) processes in TG2 (column 1) are colabeled by both DenMark (column 1) and the Syt::GFP marker (column 2). The IB is labeled by presynaptic Syt::GFP, but also has the DenMark signal (column 4). C, GFC3 (24H07-Gal4) has punctate Syt::GFP within the finger-like processes in TG3 (column 2). The IB is labeled by DenMark, with no Syt::GFP marker (column 4). GFC3 processes along the GFI axonal bend also express the DenMark label (arrowheads).

such as semaphorin-1A (Godenschwege et al., 2002; Murphey, 2003; Godenschwege and Murphey, 2009). However, in these cases, GFI axon retraction is typically only to the IB, rather than beyond the $\mathrm{CC}$, or causing complete cell loss. The molecular pathways responsible for these phenotypes may be shared with the axon retraction caused by the loss of synaptic partners, with Highwire/MYCBP2, Wallenda/DLK, and Basket/JNK as prime candidates (Ghosh et al., 2011; Borgen et al., 2017). While gap junctions play extensive roles in neuronal development (Elias and Kriegstein, 2008; Belousov and Fontes, 2013; Baker and Macagno, 2017), it is unlikely that GFI loss results from the loss of electrical coupling only, as the total removal of gap junctions from the GFI does not cause axon retraction or neuronal cell death (Blagburn et al., 1999).

The GFI axon split across the midline in response to the absence of its partner is reminiscent of sensory neuron plasticity following input deprivation (Poirier et al., 2006; Collignon et al., 2009; Rabinowitch et al., 2016) and motor circuit development changes in response to lost motor neurons (Modney and Muller, 1994; Büschges et al., 2000). This corrective rewiring could stem from either normal pathfinding and synaptogenesis or new repair pathways activated in response to unpartnered neurons. The axon split duplication with a GFI loss is different from the recent report on failed GFI pruning (Borgen et al., 2017), as the new GFI axon path is always a perfect mirror 

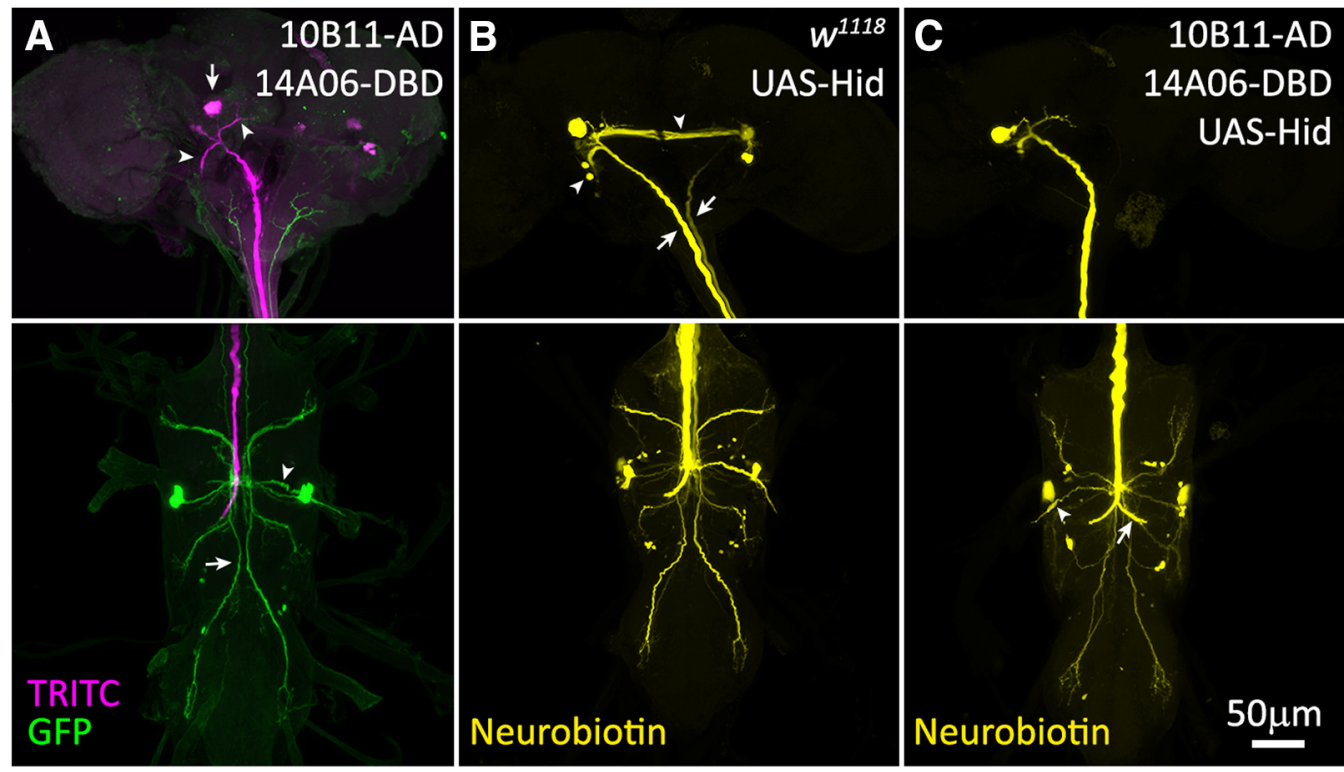

D
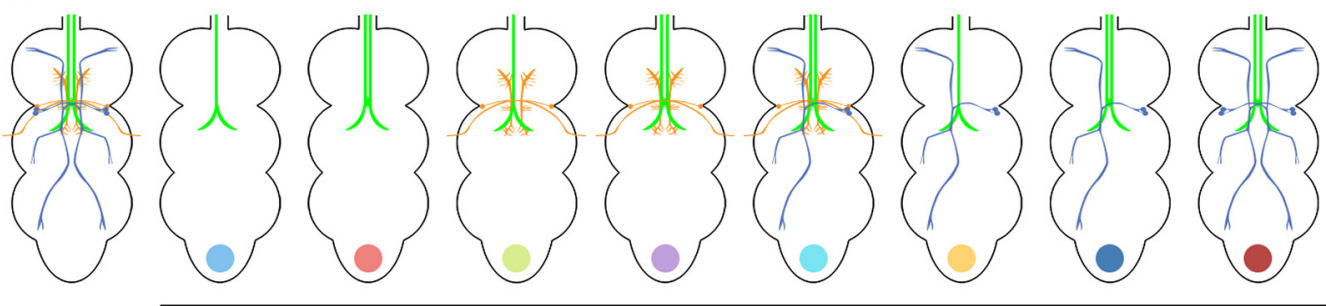

$\mathrm{Hid} /+$

Hid/10B11 $\cap$ 14A06 spGAL4

E Ablation: Full PSI and GFC1

- Retraction: $1 \mathrm{GFI}$

Ablation: Full PSI and GFC1

- Retraction: None

Ablation: GFC1 only

Retraction: $1 \mathrm{GFI}$

\section{Ablation: GFC1 Only \\ - Retraction: None}

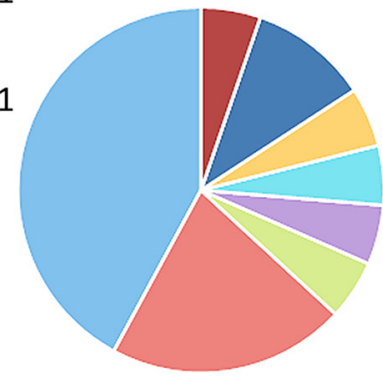

Ablation: Partial GFC1

- Retraction: None

Ablation: PSI and partial GFC1

Retraction: $1 \mathrm{GFI}$

Ablation: PSI and partial GFC1

- Retraction: None

Ablation: PSI Only

- Retraction: None

Figure 6. GFC neurons support GF circuit architectural development. A, The GFI labeled by iontophoretically injected TRITC (magenta) reveals the soma (arrow) and dendritic branches (arrowheads) in the brain (top), and descending axon in thoracic ganglion (bottom). Split Gal4 (spGal4) 10B11-AD $\cap$ 14A06-DBD drives UAS-mCD8::GFP (green) in GFC1 (bottom, arrow) and PSI (bottom, arrowhead). B, lontophoretic NB injection into the GFI (yellow) in the UAS-hid/+ control reveals the GFI (arrows) interconnected by the $\mathrm{GCl}$ (arrowheads) in the brain (top) and normal dye coupling in the thoracic ganglion (bottom). C, Driving UAS-hid with spGal4 10B11-AD $\cap 14 A 06-D B D$ results in the loss of GFC1 with occasional PSI survival (arrowhead). When GFC1 is ablated, the GCI labeling is often lost (top), one of the GFI axons is typically absent, and the remaining GFI axon always extends a compensatory contralateral axon projection (arrow). All NB injections were performed on males. $\boldsymbol{D}$, Schematic representations of GF circuit outcomes with UAS-hid/+ controls and spGal4 10B11-AD $\cap$ 14A06-DBD-driven UAS-hid cell ablation. Not pictured are instances where neither GFC1 nor PSI are ablated, and instances where both GFls are absent. $E$, Frequency of each GF circuit outcome with the targeted spGal4 10B11-AD $\cap$ 14A06-DBD-driven UAS-hid cell ablation. The pie chart color is coded to dots at the bottom of schematics in D. The sample size for UAS-hid/+ genetic controls is 21 animals, and for the spGal4 cell ablation it is 20 animals.

image of the normal axon bend, rather than an untrimmed posteriorly branched axon outgrowth. This new circuit rewiring model could be used in Drosophila genetic screens of GF circuit development (Mohr, 2014; Bassett et al., 2015; Heigwer et al., 2018) to help answer a number of important questions. Such work will be greatly aided by single-cell transgenic manipulation of presynaptic and postsynaptic neurons in the GF circuit.

In conclusion, we hope that the increase in manipulatable GFI-coupled neurons reported here will further en- 
hance this genetic model circuit. The GF circuit is ideally suited to query a wide range of important neurodevelopmental questions, including mechanisms of pathfinding, target recognition, synaptogenesis, and stabilization during neural circuit assembly and maintenance. Although the GF circuit is rightly considered one of the most straightforward and accessible Drosophila circuits, the higher degree of connectivity revealed in this study indicates a greater complexity, which is amenable to answering more in-depth questions. The large number of inputs onto, and outputs from, this model circuit provides further evidence that even the most basic circuits are deeply interconnected with the rest of the brain circuitry. As the benefits of single-cell resolution studies cannot be overstated, we hope that this enlarged GF circuit model, and the transgenic tools characterized here, will help to form part of the underpinning for future work on neural circuit dynamics.

\section{References}

Allen MJ, Drummond JA, Moffat KG (1998) Development of the giant fiber neuron of Drosophila melanogaster. J Comp Neurol 397:519531. Medline

Allen MJ, Godenschwege TA, Tanouye MA, Phelan P (2006) Making an escape: development and function of the Drosophila giant fibre system. Semin Cell Dev Biol 17:31-41. CrossRef Medline

Antonini A, Stryker MP (1993) Rapid remodeling of axonal arbors in the visual cortex. Science 260:1819-1821. Medline

Augustin H, Allen MJ, Partridge L (2011) Electrophysiological recordings from the giant fiber pathway of $D$. melanogaster. J Vis Exp 2412. CrossRef

Baker MW, Macagno ER (2017) Gap junction proteins and the wiring (rewiring) of neuronal circuits. Dev Neurobiol 77:575-586. CrossRef Medline

Bassett AR, Kong L, Liu JL (2015) A genome-wide CRISPR library for high-throughput genetic screening in Drosophila cells. J Genet Genomics 42:301-309. CrossRef Medline

Belousov AB, Fontes JD (2013) Neuronal gap junctions: making and breaking connections during development and injury. Trends Neurosci 36:227-236. CrossRef Medline

Bidaye SS, Machacek C, Wu Y, Dickson BJ (2014) Neuronal control of Drosophila walking direction. Science 344:97-101. CrossRef Medline

Blagburn JM, Alexopoulos H, Davies JA, Bacon JP (1999) Null mutation in shaking-B eliminates electrical, but not chemical, synapses in the Drosophila giant fiber system: a structural study. J Comp Neurol 404:449-458. CrossRef

Boerner J, Godenschwege TA (2011) Whole mount preparation of the adult Drosophila ventral nerve cord for giant fiber dye injection. $J$ Vis Exp 52:3080.

Borgen M, Rowland K, Boerner J, Lloyd B, Khan A, Murphey R (2017) Axon termination, pruning, and synaptogenesis in the giant fiber system of Drosophila melanogaster is promoted by highwire. Genetics 205:1229-1245. CrossRef Medline

Brand AH, Perrimon N (1993) Targeted gene expression as a means of altering cell fates and generating dominant phenotypes. Development 118:401-415. Medline

Büschges A, Djokaj S, Bässler D, Bässler U, Rathmayer W (2000) Neuromuscular plasticity in the locust after permanent removal of an excitatory motoneuron of the extensor tibiae muscle. J Neurobiol 42:148-159. Medline

Cande J, Berman GJ, Namiki S, Qiu J, Korff W, Card G, Shaevitz JW, Stern DL (2018) Optogenetic dissection of descending behavioral control in Drosophila. Elife 7:e34275.
Cardona A, Larsen C, Hartenstein V (2009) Neuronal fiber tracts connecting the brain and ventral nerve cord of the early Drosophila larva. J Comp Neurol 515:427-440. CrossRef Medline

Chen Y, Akin O, Nern A, Tsui CYK, Pecot MY, Zipursky SL (2014) Cell-type-specific labeling of synapses in vivo through synaptic tagging with recombination. Neuron 81:280-293. CrossRef Medline

Christiansen F, Zube C, Andlauer TFM, Wichmann C, Fouquet W, Owald D, Mertel S, Leiss F, Tavosanis G, Farca Luna AJ, Fiala A, Sigrist SJ (2011) Presynapses in Kenyon cell dendrites in the mushroom body calyx of Drosophila. J Neurosci 31:9696-9707. CrossRef Medline

Cohen-Cory S (2002) The developing synapse: construction and modulation of synaptic structures and circuits. Science 298:770776. CrossRef Medline

Collignon O, Voss P, Lassonde M, Lepore F (2009) Cross-modal plasticity for the spatial processing of sounds in visually deprived subjects. Exp Brain Res 192:343-358. CrossRef Medline

Crittenden JR, Skoulakis EMC, Han K-A, Kalderon D, Davis RL (1998) Tripartite mushroom body architecture revealed by antigenic markers. Learn Mem 5:38-51.

Crowley C, Spencer SD, Nishimura MC, Chen KS, Pitts-Meek S, Armaninl MP, Ling LH, McMahon SB, Shelton DL, Levinson AD, Phillips HS (1994) Mice lacking nerve growth factor display perinatal loss of sensory and sympathetic neurons yet develop basal forebrain cholinergic neurons. Cell 76:1001-1011. CrossRef

Dawydow A, Gueta R, Ljaschenko D, Ullrich S, Hermann M, Ehmann N, Gao S, Fiala A, Langenhan T, Nagel G, Kittel RJ (2014) Channelrhodopsin-2-XXL, a powerful optogenetic tool for low-light applications. Proc Natl Acad Sci U S A 111:13972-13977. CrossRef Medline

Dionne H, Hibbard KL, Cavallaro A, Kao JC, Rubin GM (2018) Genetic reagents for making split-GAL4 lines in Drosophila. Genetics 209:31-35. CrossRef Medline

Elias LAB, Kriegstein AR (2008) Gap junctions: multifaceted regulators of embryonic cortical development. Trends Neurosci 31:243250. CrossRef Medline

Enneking E-M, Kudumala SR, Moreno E, Stephan R, Boerner J, Godenschwege TA, Pielage J (2013) Transsynaptic coordination of synaptic growth, function, and stability by the L1-type CAM neuroglian. PLoS Biol 11:e1001537. CrossRef Medline

Fayyazuddin A, Zaheer MA, Hiesinger PR, Bellen HJ (2006) The nicotinic acetylcholine receptor Dalpha7 is required for an escape behavior in Drosophila. PLoS Biol 4:e63.

Fotowat H, Fayyazuddin A, Bellen HJ, Gabbiani F (2009) A novel neuronal pathway for visually guided escape in Drosophila melanogaster. J Neurophysiol 102:875-885. CrossRef Medline

Frank DD, Jouandet GC, Kearney PJ, MacPherson LJ, Gallio M (2015) Temperature representation in the Drosophila brain. Nature 519:358-361. CrossRef Medline

Gaudry Q, Kristan WB (2009) Behavioral choice by presynaptic inhibition of tactile sensory terminals. Nat Neurosci 12:1450-1457. CrossRef Medline

Ghosh AS, Wang B, Pozniak CD, Chen M, Watts RJ, Lewcock JW (2011) DLK induces developmental neuronal degeneration via selective regulation of proapoptotic JNK activity. J Cell Biol 194:751764. CrossRef Medline

Gibson DA, Ma L (2011) Developmental regulation of axon branching in the vertebrate nervous system. Development 138:183-195. CrossRef Medline

Godenschwege T, Murphey R (2009) Genetic interaction of neuroglian and Semaphorin1a during guidance and synapse formation. J Neurogenet 23:147-155. CrossRef Medline

Godenschwege TA, Hu H, Shan-Crofts X, Goodman CS, Murphey RK (2002) Bi-directional signaling by Semaphorin 1a during central synapse formation in Drosophila. Nat Neurosci 5:1294-1301. CrossRef Medline

Gorin PD, Johnson EM (1979) Experimental autoimmune model of nerve growth factor deprivation: effects on developing peripheral 
sympathetic and sensory neurons. Proc Natl Acad Sci U S A 76:5382-5386. Medline

Hanganu IL, Okabe A, Lessmann V, Luhmann HJ (2009) Cellular mechanisms of subplate-driven and cholinergic input-dependent network activity in the neonatal rat somatosensory cortex. Cereb Cortex 19:89-105. CrossRef

Heigwer F, Port F, Boutros M (2018) RNA interference (RNAi) screening in Drosophila. Genetics 208:853-874. CrossRef Medline

Hsu CT, Bhandawat V (2016) Organization of descending neurons in Drosophila melanogaster. Sci Rep 6:20259. CrossRef

Huang Q, Zhou D, DiFiglia M (1992) Neurobiotin, a useful neuroanatomical tracer for in vivo anterograde, retrograde and transneuronal tract-tracing and for in vitro labeling of neurons. J Neurosci Methods 41:31-43. CrossRef

Hubel DH, Wiesel TN (1970) The period of susceptibility to the physiological effects of unilateral eye closure in kittens. J Physiol 206:419-436. Medline

Hubel DH, Wiesel TN, LeVay S (1977) Plasticity of ocular dominance columns in monkey striate cortex. Philos Trans R Soc Lond B Biol Sci 278:377-409. Medline

Jacobs K, Todman MG, Allen MJ, Davies JA, Bacon JP (2000) Synaptogenesis in the giant-fibre system of Drosophila: interaction of the giant fibre and its major motorneuronal target. Development 127:5203-5212. Medline

Jenett A, et al. (2012) A gal4-driver line resource for Drosophila neurobiology. Cell Rep 2:991-1001. CrossRef Medline

Joseph RM, Sun JS, Tam E, Carlson JR (2017) A receptor and neuron that activate a circuit limiting sucrose consumption. Elife 6:e24992.

Kanca O, Bellen HJ, Schnorrer F (2017) Gene tagging strategies to assess protein expression, localization, and function in Drosophila. Genetics 207:389-412. Medline

Kandler K, Katz LC (1995) Neuronal coupling and uncoupling in the developing nervous system. Curr Opin Neurobiol 5:98-105. Medline

Kennedy T, Broadie K (2017) Fragile X mental retardation protein restricts small dye iontophoresis entry into central neurons. J Neurosci 37:9844-985. CrossRef

King DG, Wyman RJ (1980) Anatomy of the giant fibre pathway in Drosophila. I. Three thoracic components of the pathway. J Neurocytol 9:753-770. Medline

Lin TY, Luo J, Shinomiya K, Ting CY, Lu Z, Meinertzhagen IA, Lee CH (2016) Mapping chromatic pathways in the Drosophila visual system. J Comp Neurol 524:213-227. CrossRef Medline

Luan H, Peabody NC, Vinson CR, White BH (2006) Refined spatial manipulation of neuronal function by combinatorial restriction of transgene expression. Neuron 52:425-436. CrossRef Medline

Maher BJ, McGinley MJ, Westbrook GL (2009) Experiencedependent maturation of the glomerular microcircuit. Proc Natl Acad Sci U S A 106:16865-16870. CrossRef Medline

Martinez VG, Javadi CS, Ngo E, Ngo L, Lagow RD, Zhang B (2007) Age-related changes in climbing behavior and neural circuit physiology in drosophila. Dev Neurobiol 67:778-791. CrossRef Medline

Modney B, Muller KJ (1994) Novel synapses compensate for a neuron ablated in embryos. Proc Biol Sci 257:263-269. CrossRef Medline

Mohr SE (2014) RNAi screening in Drosophila cells and in vivo. Methods 68:82-88. CrossRef Medline

Murphey RK (2003) Targeted expression of shibire ts and semaphorin 1a reveals critical periods for synapse formation in the giant fiber of Drosophila. Development 130:3671-3682. Medline

Muthukumar AK, Stork T, Freeman MR (2014) Activity-dependent regulation of astrocyte GAT levels during synaptogenesis. Nat Neurosci 17:1340-1350. CrossRef Medline

Nagel G, Brauner M, Liewald JF, Adeishvili N, Bamberg E, Gottschalk A (2005) Light activation of Channelrhodopsin-2 in excitable cells of caenorhabditis elegans triggers rapid behavioral responses. Curr Biol 15:2279-84
Namiki S, Dickinson MH, Wong AM, Korff W, Card GM (2018) The functional organization of descending sensory-motor pathways in Drosophila. Elife 7:50.

Nern A, Pfeiffer BD, Rubin GM (2015) Optimized tools for multicolor stochastic labeling reveal diverse stereotyped cell arrangements in the fly visual system. Proc Natl Acad Sci U S A 112:E2967-E2976. CrossRef

Nicolai LJJ, Ramaekers A, Raemaekers T, Drozdzecki A, Mauss AS, Yan J, Landgraf M, Annaert W, Hassan BA (2010) Genetically encoded dendritic marker sheds light on neuronal connectivity in Drosophila. Proc Natl Acad Sci U S A 107:20553-20558. CrossRef Otsuna H, Ito M, Kawase T (2018) Color depth MIP mask search: a new tool to expedite Split-GAL4 creation. https://www.biorxiv.org/ content/early/2018/05/09/318006.

Pearson HE, Stoffler DJ (1992) Retinal ganglion cell degeneration following loss of postsynaptic target neurons in the dorsal lateral geniculate nucleus of the adult cat. Exp Neurol 116:163-171. Medline

Pézier A, Jezzini SH, Marie B, Blagburn JM (2014) Engrailed alters the specificity of synaptic connections of Drosophila auditory neurons with the giant fiber. J Neurosci 34:11691-11704. CrossRef

Pfeiffer BD, Ngo TTB, Hibbard KL, Murphy C, Jenett A, Truman JW, Rubin GM (2010) Refinement of tools for targeted gene expression in Drosophila. Genetics 186:735-755. CrossRef Medline

Phelan P (2005) Innexins: members of an evolutionarily conserved family of gap-junction proteins. Biochim Biophys Acta 1711:225245. CrossRef Medline

Phelan P, Bacon P, Moffat KG, Kane CJO, Davies JA (1996) Mutations in shaking-B prevent electrical synapse formation in the Drosophila giant fiber system. J Neurosci 16:1101-1113. CrossRef

Phelan P, Goulding LA, Tam JLY, Allen MJ, Dawber RJ, Davies JA, Bacon JP (2008) Molecular mechanism of rectification at identified electrical synapses in the Drosophila giant fiber system. Curr Biol 18:1955-1960. CrossRef

Poirier C, Collignon O, Scheiber C, Renier L, Vanlierde A, Tranduy D, Veraart C, De Volder AG (2006) Auditory motion perception activates visual motion areas in early blind subjects. Neuroimage 31:279-285. CrossRef Medline

Power ME (1948) The thoracico-abdominal nervous system of an adult insect, Drosophila melanogaster. J Comp Neurol 88:347409. Medline

Rabinowitch I, Laurent P, Zhao B, Walker D, Beets I, Schoofs L, Bai J, Schafer WR, Treinin M (2016) Neuropeptide-driven cross-modal plasticity following sensory loss in Caenorhabditis elegans. PLoS Biol 14:e1002348. CrossRef

Schindelin J, Arganda-Carreras I, Frise E, Kaynig V, Longair M, Pietzsch T, Preibisch S, Rueden C, Saalfeld S, Schmid B, Tinevez J-Y, White DJ, Hartenstein V, Eliceiri K, Tomancak P, Cardona A (2012) Fiji: an open-source platform for biological-image analysis. Nat Methods 9:676-682. CrossRef Medline

Schneider CA, Rasband WS, Eliceiri KW (2012) NIH Image to ImageJ: 25 years of image analysis. Nat Methods 9:671-675. Medline

Sivaguru M, Urban MA, Fried G, Wesseln CJ, Mander L, Punyasena SW (2016) Comparative performance of airyscan and structured illumination superresolution microscopy in the study of the surface texture and 3D shape of pollen. Microsc Res Tech 81:101-114. CrossRef

Smith HK, Roberts IJH, Allen MJ, Connolly JB, Moffat KG, O'Kane CJ (1996) Inducible ternary control of transgene expression and cell ablation in Drosophila. Dev Genes Evol 206:14-24. CrossRef Medline

Staudt T, Lang MC, Medda R, Engelhardt J, Hell SW (2007) 2,2'Thiodiethanol: a new water soluble mounting medium for high resolution optical microscopy. Microsc Res Tech 70:1-9. CrossRef Medline

Stebbings LA, Todman MG, Phillips R, Greer CE, Tam J, Phelan P, Jacobs K, Bacon JP, Davies JA (2002) Gap junctions in Drosophila: developmental expression of the entire innexin gene family. Mech Dev 113:197-205. Medline 
Stensmyr MC, Dweck HKM, Farhan A, Ibba I, Strutz A, Mukunda L, Linz J, Grabe V, Steck K, Lavista-Llanos S, Wicher D, Sachse S, Knaden M, Becher PG, Seki Y, Hansson BS (2012) A conserved dedicated olfactory circuit for detecting harmful microbes in drosophila. Cell 151:1345-1357. CrossRef

Sun Y, Wyman RJ (1997) Neurons of the Drosophila giant fiber system: I. Dorsal longitudinal motor neurons. J Comp Neurol 387:157-166. Medline

Talay M, Richman EB, Snell NJ, Hartmann GG, Fisher JD, Sorkaç A, Santoyo JF, Chou-Freed C, Nair N, Johnson M, Szymanski JR, Barnea G (2017) Transsynaptic mapping of second-order taste neurons in flies by trans-tango. Neuron 96:783-795.e4.

Tanouye MA, Wyman RJ (1980) Motor outputs of giant nerve fiber in Drosophila. J Neurophysiol 44:405-421. CrossRef Medline

Thomas JB, Wyman RJ (1984) Mutations altering synaptic connectivity between identified neurons in Drosophila. J Neurosci 4:530538. Medline

Tirian L, Dickson B (2017) The VT GAL4, LexA, and split-GAL4 driver line collections for targeted expression in the Drosophila nervous system. https://www.biorxiv.org/content/biorxiv/early/2017/10/05/ 198648.full.pdf.

Tosches MA (2017) Developmental and genetic mechanisms of neural circuit evolution. Dev Biol 431:16-25. CrossRef

Trimarchi JR, Schneiderman AM (1993) Giant fiber activation of an intrinsic muscle in the mesothoracic leg of Drosophila melanogaster. J Exp Biol 177:149-167. Medline

Trimarchi JR, Schneiderman AM (1995) Different neural pathways coordinate Drosophila flight initiations evoked by visual and olfactory stimuli. J Exp Biol 198:1099-1104. Medline
Trimarchi JR, Jin P, Murphey RK (1999) Controlling the motor neuron. Int Rev Neurobiol 43:241-264. Medline

Uesaka N (2005) Activity dependence of cortical axon branch formation: a morphological and electrophysiological study using organotypic slice cultures. J Neurosci 25:1-9. CrossRef Medline

von Reyn CR, Breads P, Peek MY, Zheng GZ, Williamson WR, Yee AL, Leonardo A, Card GM (2014) A spike-timing mechanism for action selection. Nat Neurosci 17:962-970. CrossRef Medline

Ward A, Hong W, Favaloro V, Luo L (2015) Toll receptors instruct axon and dendrite targeting and participate in synaptic partner matching in a Drosophila olfactory circuit. Neuron 85:1013-1028. CrossRef

Zhang YQ, Rodesch CK, Broadie K (2002) Living synaptic vesicle marker: synaptotagmin-GFP. Genesis 34:142-145. CrossRef Medline

Zheng Z, Lauritzen JS, Perlman E, Robinson CG, Nichols M, Milkie D, Torrens O, Price J, Fisher CB, Sharifi N, Calle-Schuler SA, Kmecova L, Ali IJ, Karsh B, Trautman ET, Bogovic JA, Hanslovsky P, Jefferis GSXE, Kazhdan M, Khairy K, et al. (2018) A complete electron microscopy volume of the brain of adult Drosophila melanogaster. Cell 174:730-743.e2.

Zhou L, Schnitzler A, Agapite J, Schwartz LM, Steller H, Nambu JR (1997) Cooperative functions of the reaper and head involution defective genes in the programmed cell death of Drosophila central nervous system midline cells. Proc Natl Acad Sci U S A 94:5131-5136. Medline 